\title{
Multi-mechanical waves against Alzheimer's disease pathology: a systematic review
}

\author{
Francisca Monteiro ${ }^{1,3^{*}+}$ (D) loannis Sotiropoulos ${ }^{2,3,4 \dagger}$, Óscar Carvalho ${ }^{1}$, Nuno Sousa ${ }^{2,3}$ and Filipe S. Silva ${ }^{1}$
}

\begin{abstract}
Alzheimer's disease (AD) is the most common cause of dementia, affecting approximately 40 million people worldwide. The ineffectiveness of the available pharmacological treatments against AD has fostered researchers to focus on alternative strategies to overcome this challenge. Mechanical vibrations delivered in different stimulation modes have been associated with marked improvements in cognitive and physical performance in both demented and nondemented elderly. Some of the mechanical-based stimulation modalities in efforts are earlier whole-body vibration, transcranial ultrasound stimulation with microbubble injection, and more recently, auditory stimulation. However, there is a huge variety of treatment specifications, and in many cases, conflicting results are reported. In this review, a search on Scopus, PubMed, and Web of Science databases was performed, resulting in 37 papers. These studies suggest that mechanical vibrations delivered through different stimulation modes are effective in attenuating many parameters of AD pathology including functional connectivity and neuronal circuit integrity deficits in the brains of AD patients, as well as in subjects with cognitive decline and non-demented older adults. Despite the evolving preclinical and clinical evidence on these therapeutic modalities, their translation into clinical practice is not consolidated yet. Thus, this comprehensive and critical systematic review aims to address the most important gaps in the reviewed protocols and propose optimal regimens for future clinical application.
\end{abstract}

Keywords: Alzheimer's disease, Auditory stimulation, Mechanical vibrations, Ultrasounds, Whole-body vibration

\section{Introduction}

Alzheimer's disease (AD) is an age-related neurodegenerative disorder characterized by progressive structural and functional lesions in the brain, which include neuronal atrophy and synaptic loss accompanied by neuronal death, brain network damage, and aberrant network oscillations [1]. These brain lesions are correlated with the accumulation and aggregation of amyloid $\beta(\mathrm{A} \beta)$ peptide and Tau protein [2-4], resulting in extracellular $A \beta$ plaques and intracellular neurofibrillary tangles of hyperphosphorylated Tau protein in the AD brain $[5,6]$. This

\footnotetext{
*Correspondence: id8700@alunos.uminho.pt

${ }^{\dagger}$ Francisca Monteiro and loannis Sotiropoulos contributed equally to this work.

${ }^{1}$ Center for Microelectromechanical Systems (CMEMS), University of Minho, Campus Azurém, 4800-058 Guimarães, Portugal

Full list of author information is available at the end of the article
}

disorder is clinically characterized by evolving memory impairments followed by broad cognitive decline and brain degeneration that ultimately leads to death. $\mathrm{AD}$ appears to be a devastating disorder that affects approximately 40 million people worldwide while this number is expected to be triplicated by $2050[7,8]$. Based on its increasing incidence and high societal impact, the World Health Organization has declared $\mathrm{AD}$ and dementia a major priority health issue [8]. However, besides the extensive efforts over years to find a pharmacological treatment for $\mathrm{AD}$, there is no effective therapy that can stop or revert brain pathology as well as cognitive and other behavioral deficits of AD [9].

Recently, alternative therapeutic strategies (e.g. electrical-, magnetic-, optical- and mechanical-based therapies) for $A D$ have been intensively investigated [10-12]. Among the most promising ones, the mechanical wavebased modalities acting at different vibration modes have

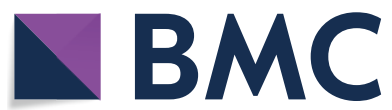

(c) The Author(s) 2021. Open Access This article is licensed under a Creative Commons Attribution 4.0 International License, which permits use, sharing, adaptation, distribution and reproduction in any medium or format, as long as you give appropriate credit to the original author(s) and the source, provide a link to the Creative Commons licence, and indicate if changes were made. The images or other third party material in this article are included in the article's Creative Commons licence, unless indicated otherwise in a credit line to the material. If material is not included in the article's Creative Commons licence and your intended use is not permitted by statutory regulation or exceeds the permitted use, you will need to obtain permission directly from the copyright holder. To view a copy of this licence, visit http://creativecommons.org/licenses/by/4.0/. The Creative Commons Public Domain Dedication waiver (http://creativeco mmons.org/publicdomain/zero/1.0/) applies to the data made available in this article, unless otherwise stated in a credit line to the data. 
been associated with improvement of AD neuropathology beyond the reduction of $A \beta$ and Tau accumulation, affecting various pathological parameters involved in $\mathrm{AD}$ such as chronic neuroinflammation, synaptic loss, and neuronal atrophy; importantly, these alterations are also accompanied by an attenuation of cognitive and other behavioral deficits. These modalities are non-invasive, low-cost, and easily customized brain stimulation techniques that include whole-body vibration (WBV), transcranial ultrasound stimulation (TUSS), and auditory stimulation (AS). The WBV training consists of passive exercise training delivered by a vibratory platform [13, 14] and has been associated with numerous beneficial effects on neuromuscular function [15-17], vascular diseases [18], and neurological conditions [19, 20] in older adults. During the last five years, a few studies have tested WBV in demented or AD patients, showing promising results $[21,22]$. Moreover, the use of TUSS, another mechanical-based modality, in experimental animals has also provided some solid evidence on its beneficial effects on brain plasticity and function as well as neuronal circuit integrity in young wild-type animals [23-29]. More recent studies support the use of the AS, a sensory-based stimulation strategy delivered by trains of tones, as a potential solution to entrain gamma oscillation in the brains of $A D$ transgenic mice, which has been shown to reduce $\mathrm{AD}$ neuropathology and behavioral impairment $[30,31]$.

These mechanical-based therapeutic modalities are in the early exploration of alternative therapies to attenuateAD pathology and associated behavioral deficits. Although they share the same fundamental energy-transfer carrier (which is the mechanical waves), the stimulation modalities require different application protocols and acoustic/mechanical parameters, and therefore distinct specifications must be considered. In addition, there is a huge variety of treatment specifications within the same stimulation protocols, and in many cases, conflicting results are reported. Thus, this systematic review aims to evaluate and critically discuss the existing evidence on these multi-mechanical wave-based stimuli and their impact on different aspects of AD pathology, but also on age-related impairments that often occur concomitantly with the development of $\mathrm{AD}$ and related dementia (e.g., impairments in neuromuscular function and mobility).

\section{Methods}

This systematic review was conducted according to the Preferred Reporting Items for Systematic Reviews and Meta-Analyses (PRISMA) guidelines.

\section{Search strategy}

The electronic search was performed in Scopus, PubMed, and Web of Science databases by the date of 26 December 2020. The following search terms were employed: ("Alzheimer's disease" OR "cognitive decline" OR "mild cognitive impairment") AND ("music therapy" OR "acoustic wave" OR "acoustic stimulation" OR "auditory stimulation" OR "sound stimulation" OR "somatosensory stimulation" OR "whole-body vibration" OR "vibration therapy" OR "sound wave" OR "mechanical wave" OR "rhythmic sensory stimulation" OR "vibroacoustic therapy" OR "vibrotactile stimulation" OR "physioacoustic therapy" OR "binaural beats" OR "tone stimulation" OR "scanning ultrasound" OR "focused ultrasound" OR "sonication" OR "ultrasonic therapy").

\section{Eligibility criteria}

The established requirements for eligibility are depicted in Table 1. Titles and abstracts were screened and filtered according to the criteria.

\section{Quality assessment}

In line with previous systematic reviews in $\mathrm{AD}$ [32, 33], the quality assessment of the reviewed clinical studies was performed using the Effective Public Health Practice Project (EPHPP) Quality Assessment Tool, and classified according to their global rating as having low (no "Weak" ratings), moderate (one "Weak" rating) or high (two or more "Weak" ratings) risk of bias [34]. For

Table 1 Eligibility criteria for admission in this review

\begin{tabular}{|c|c|}
\hline Inclusion criteria & Exclusion criteria \\
\hline $\begin{array}{l}\text { Preclinical and clinical studies reporting noninvasive application of } \\
\text { mechanical vibration-based approaches to impact AD pathology and/or } \\
\text { related symptoms } \\
\text { Preclinical and clinical studies reporting the application of mechanical } \\
\text { vibration-based approaches to impact physical and/or cognitive deficits in } \\
\text { aged animal models or non-demented older adults (aged over } 60 \text { years) } \\
\text { Studies written in English }\end{array}$ & $\begin{array}{l}\text { Reviews, conference papers, proceedings papers, editorials, and surveys } \\
\text { Studies reporting the use of mechanical waves to facilitate/interfere with } \\
\text { drug/gene delivery into the brain } \\
\text { Studies in which mechanical stimulation is combined with other (unusual) } \\
\text { interventions/activities (e.g., physical exercise) } \\
\text { Studies in which nonperiodic mechanical waves are used to stimulate the } \\
\text { brain } \\
\text { Studies that evaluate the effect of mechanical stimulation in individuals } \\
\text { suffering from cognitive decline derived from other diseases/conditions } \\
\text { (e.g., stroke, ischemia, Parkinson's disease) }\end{array}$ \\
\hline
\end{tabular}


animal studies, the Stroke Therapy Academic Industry Roundtable (STAIR) preclinical recommendations were used [35]. Each study was assessed independently by two authors (FM and OC) and, when different results were achieved, the authors reevaluated the original paper until reaching a consensus. Complete quality assessment data, including the defined criteria, the results for each study, and their categorization, are provided as Additional file 1.

\section{Results}

\section{Article selection and quality assessment}

The electronic literature search resulted in 1860 publications, and other 14 papers found in the literature review were added to the study list; then 649 duplicates were removed. By screening the titles and abstracts of the remaining 1225 titles, 168 full-text articles were assessed for eligibility, and 37 of them met the defined criteria and were included in the current systematic review. The article selection process is schematized in Fig. 1.

Concerning the methodological quality assessment, the EPHPP Quality Assessment Tool classified 17 clinical studies $\left(n_{\mathrm{WBV}}=10, n_{\mathrm{TUSS}}=3, n_{\mathrm{AS}}=4\right)$ as having low risk of bias, three WBV and one TUSS studies were classified as having a moderate risk of bias, and only two studies presented a high risk of bias (two WBV papers). For the animal studies, the STAIR preclinical recommendations showed that the overall quality was not satisfactory; none of the included studies performed a sample size calculation, only one correctly described the method

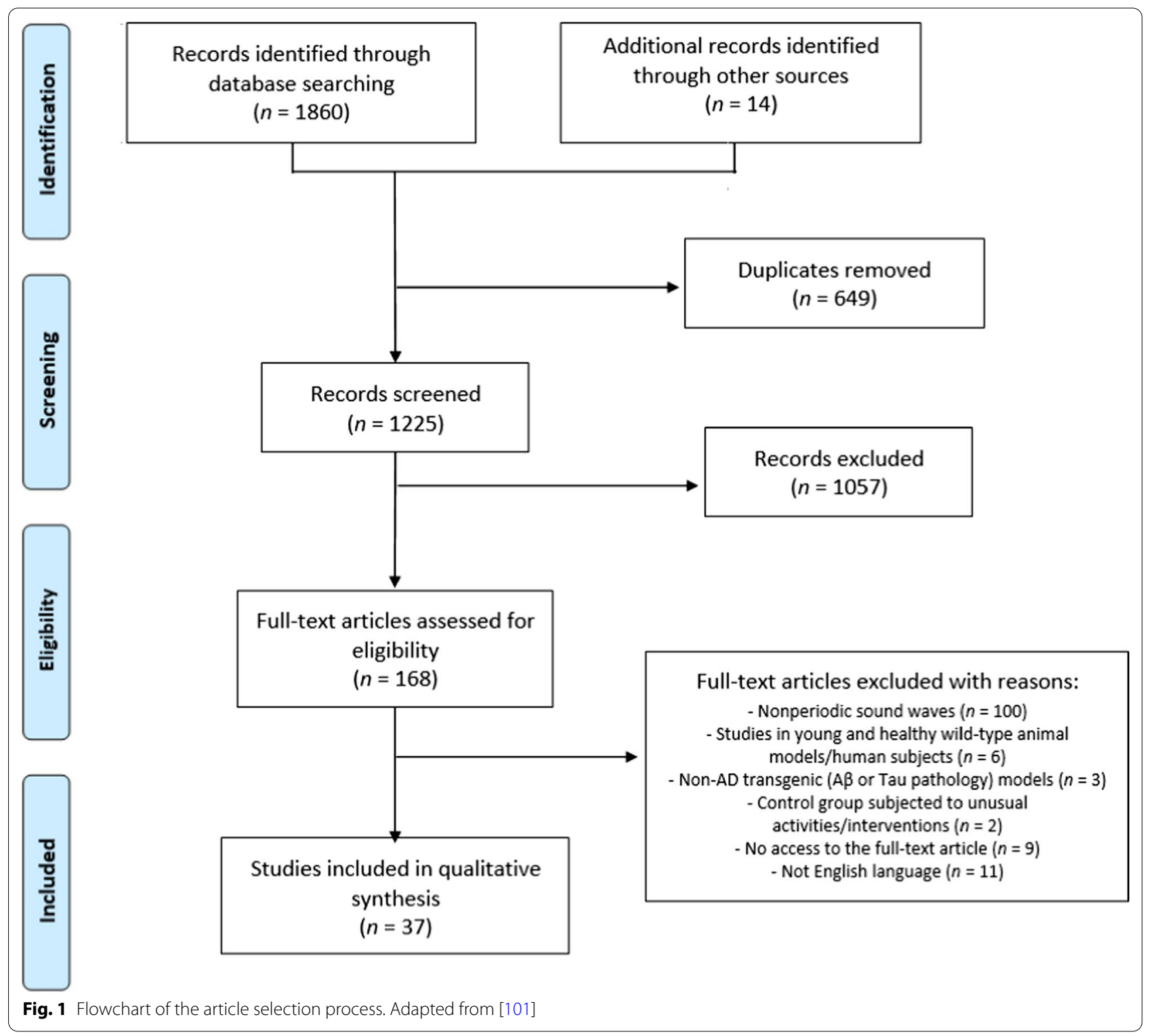


of allocation concealment [36], only two studies reported the reasons for animal exclusion from analysis [37, 38], and the majority of them did not mention the existence of any exclusion at all. Although many studies state that the animals were randomly allocated to the experimental groups, only one study provided details about the method of randomization [37]. The overall quality assessment data are presented in Additional file 1: Tables S1 and $\mathrm{S} 2$.

\section{Profile of the included articles}

This systematic review focuses on both preclinical and clinical studies that used three types of mechanical vibration-based stimulation: WBV $(n=14$, Table 2$)$, TUSS $(n=17$, Table 3$)$, and AS $(n=6$, Table 4$)$.

At the clinical level, the effect of WBV training was assessed in cohorts of non-demented older adults $(n=11)$ or individuals diagnosed with mild or moderate dementia $(n=3)$ using different cognitive status tests such as Cantonese mini-mental state examination and Mini-Mental State Examination.

The TUSS and AS studies included in this systematic review describe both preclinical and clinical evidence. Regarding the 17 TUSS studies, five of them were dedicated to the investigation of the effectiveness of combined ultrasound treatment and microbubble injection in AD patients; the remaining TUSS studies were conducted in AD transgenic mouse models $(n=11)$ and aged amyloid- $\beta(\mathrm{A} \beta)$-positive dogs $(n=1)$.

For AS papers, two animal studies were performed in $\mathrm{AD}$ transgenic mice using periodic stimuli while one of them combined AS with visual stimulation. The remaining four AS studies were performed in humans using heterogeneous protocols; one study provided vibroacoustic stimulation (i.e., therapeutic strategy using vibratory sound stimuli) to AD patients; another study tested the effect of combined auditory and visual stimuli in AD patients, and the other two studies described the implementation of an AS protocol during sleep in amnestic mild cognitive impairment (MCI) patients (i.e. a prodromal stage of AD) and healthy non-demented older subjects.

The data collected from the included studies are organized in Tables 2, 3 and 4, summarizing the implemented protocols and the most important findings from the WBV, TUSS, and AS studies, respectively. The studies are compiled in chronological order, starting with animal studies, followed by clinical trials. The readouts used in the original papers are categorized and compared in Table 5.

Basic principles of the three mechanical-based modalities and their relationship to brain pathology

WBV training consists of passive exercise training delivered by a vibratory platform in which high-frequency
(5-40 Hz) and small-amplitude mechanical stimuli are applied to the entire body [13, 14]. During WBV training, the vibration delivered by the oscillating platform stimulates the neuromuscular system by activating muscles through spinal reflexes and increasing synchronous motor unit recruitment [39]. These phenomena enhance neuromuscular excitability, inducing muscle contractions $[39,40]$, which has been shown to produce numerous beneficial effects in older adults on neuromuscular function [15-17], vascular diseases [18], and neurological conditions $[19,20]$. However, despite the wide range of clinical application, limited doses of WBV are well tolerated by the elderly, as overexposure to WBV training can result in over-stress injuries [14]. On the other hand, insufficient stimulation may result in the lack of the desired improvement. For this reason, the dose-response relationship for WBV needs to be carefully defined for each application or individual (including an AD patienttailored procedure), and no optimal protocols have yet been established.

TUSS is a modality used in combination with microbubble injection, which transiently opens the bloodbrain barrier (BBB). After intravenous injection in the brain, the ultrasound (US)-sonicated microbubbles undergo expansion-contraction cycles that disrupt the tight junctions along the blood vessel walls, creating new entries through the BBB [41], usually reversed within $24 \mathrm{~h}$. Importantly, the size and dose of microbubbles, as well as their manipulation and synchronization with sonication must be appropriate to allow the efficiency and success of the process. Also, ultrasonic waves are mainly characterized via the center frequency (associated with the energy carried by the waves), the emission mode (i.e., continuous or pulsed mode), and the power density or pressure that is applied to a certain body/surface (associated with the intensity of the stimulation). The TUSS microbubble-assisted approach has been widely used in the last decade to facilitate the delivery of therapeutic agents for the treatment of a wide range of neurological disorders, showing promising efficiency to facilitate their pharmacological effect [42-45]. However, recent studies have shown the beneficial action of TUSS (i.e. without association to any drug) to stimulate neurogenesis, neuronal excitability, and/or brain network activity in wild-type experimental animals [23-29] and improve AD brain pathology and related memory deficits in AD mouse models [23-29, 46, 47]. These achievements have sparked clinical interest in the pursuit of an effective noninvasive intervention based on the transcranial application of US to ameliorate AD pathology. Yet, the novelty of this technique entails many doubts concerning the optimal 
protocols and the long-term effects as discussed below (see Section Limitations and future directions).

More recently, AS has been receiving increased attention due to its effectiveness in reducing $A \beta$ and Tau pathology and improving cognitive abilities in $\mathrm{AD}$ animal models [30, 48]. This is a sensory-based stimulation strategy delivered by trains of tones repeated at a specific frequency which has been associated with marked entrainment of brainwaves in the brains of AD transgenic mice $[30,48]$ and $\mathrm{AD}$ patients $[31,49]$. In fact, $\mathrm{A} \beta$ and Tau pathologies are known to alter brain rhythmic oscillatory activities [50], mainly in high-frequency band waves, impacting the high-order cognitive domains [51, 52]. High-frequency brainwaves, e.g. gamma oscillations $(30-100 \mathrm{~Hz})$, are disrupted in both AD patients and AD transgenic mouse models [51, 53]. During the last years, sensory stimulation has been used to affect/stimulate gamma band oscillations in the brain. Importantly, this brainwave entrainment has been correlated with the attenuation of $\mathrm{AD}$ pathomechanisms in $\mathrm{AD}$ mouse models [51, 54], as well as with the improvement of cognitive function and sleep disturbances in younger adults [55].

\section{Impact on $\mathrm{AD}$ pathomechanisms and associated brain malfunction}

This section presents and discusses findings from studies on the three modalities (Tables 2, 3, 4), classified into cellular, molecular, brain function/connectivity and behavioral outputs.

\section{$A \beta$ and Tau pathologies}

In WBV studies, no biochemical or brain imaging analysis was performed to assess possible alterations of $A \beta$ and/or Tau pathology in the brains of participants. On the other hand, the reviewed studies on TUSS and AS provide plenty of preclinical evidence on their effectiveness to reduce/block $A \beta$ and Tau accumulation and aggregation.

TUSS Ultrasound pulses delivered transcranially inhibit the formation of $\mathrm{A} \beta$ plaques by decreasing soluble $A \beta$ species, as shown in the brains of three different $\mathrm{AD}$ transgenic mouse models with advanced $\mathrm{A} \beta$ pathology, i.e. APP23, 3xTg, and 5xFAD [36, 56-58]. In some cases, pulsed TUSS is able to markedly reduce $\mathrm{A} \beta$ plaque load (affecting number, size, area) in different $\mathrm{AD}$ transgenic mice after single $[37,59]$ or multiple US sessions [36, 37, 57, 58, 60, 61]. It should be noted that these studies have typically used similar stimulation frequencies (center frequency $1 \mathrm{MHz}$, pulsing at $1-10 \mathrm{~Hz}$ ), but very discrepant duration (few minutes to $1 \mathrm{~h}$ for each session, delivered either in a single application or over several weeks). Moreover, two stud- ies in A $\beta$-based transgenic mice (i.e., TgCRND8) with abundant amyloid plaque load reported that part of the TUSS beneficial effect was lost when follow-up periods were considered; both Jordão et al. (2013) and Poon et al. (2018) reported that the reduction of $A \beta$ plaque area was observed four to seven days after mouse brain stimulation, but this recovery was lost two weeks after a single session of $2 \mathrm{~min}$ [37] or $8 \mathrm{~min}$ [59]. Another study in aged $A \beta$-positive dogs showed a tendency for reduced $A \beta$ load in the prefrontal cortex of the US-treated hemisphere, in both single- and multiple-session interventions [62]. This limited impact of TUSS treatment on $\mathrm{A} \beta$ load could be attributed to the insufficient stimulus duration or intensity (such as insufficient duration of each stimulation, e.g. 2 min stimulation once a week, 4 weeks period, or insufficient ultrasonic intensity, e.g. $0.28 \mathrm{MHz}$ pulsing at $1 \mathrm{~Hz}$, as the center frequency is relatively lower compared to all the other preclinical experiments in $\mathrm{AD}$ transgenic mice). Another parameter to be considered is the advanced state of $A \beta$ pathology of the experimental animals (dogs in the above study), which may suggest that such applications should be more beneficial if they are offered in early stages of AD brain pathology [62].

At the clinical level, only two studies have investigated the effect of TUSS on $A \beta$ in the brains of mildto-moderate $\mathrm{AD}$ patients (aged $57-73$ years), by using $\left[{ }^{18} \mathrm{~F}\right]$-florbetaben PET scan [63, 64]. However, conflicting outcomes have been reported. The first study by Lipsman and colleagues (2018) found no significant alterations in $\mathrm{A} \beta$ level after TUSS treatment $(7.5 \mathrm{~min}$ once a week, 3 months period) delivered using a helmet-like device (whole-brain stimulation) in mild-tomoderate AD patients [63]. On the contrary, D'Haese et al. (2020) reported a reduction of $A \beta$ plaques in the hippocampus and entorhinal cortex of the treated hemisphere after unilateral stimulation (3 sessions, 6 weeks period) in patients at early stage of $\mathrm{AD}$ and at a similar age [65].

Although less studied, the beneficial effect of TUSS on Tau hyperphosphorylation and aggregation has recently emerged. Experimental studies have described a TUSSdriven decrease of $\mathrm{p}$-Tau levels in the hippocampus of three different mutant-Tau transgenic mouse models (i.e., $\mathrm{K} 3, \mathrm{rTg} 4510$, and $3 \mathrm{xTg}$-AD mice) after single $[45,58]$ or multiple US sonications ( 2.4 min once a week, 15 weeks period) [66]. Reduction is also observed for neurofibrillary tangles, the final intraneuronal aggregates of Tau, in K3 mice [66]. Interestingly, Karakatsani et al. (2019) observed a reduction of $\mathrm{p}$-Tau in both hemispheres of 4-month-old rTg4510 mouse brain after a single session of unilateral-target US sonication [45], showing that US is capable of producing a widespread and systemic 
response across the brain beyond the sonicated areas, even with a minimum dose of TUSS (1-min session).

AS For AS, only preclinical studies have monitored its effect on $A \beta$ load, using trains of tones repeating at $40 \mathrm{~Hz}$. In the study conducted by Martorell et al. (2019), the trains of acoustic tones $(1 \mathrm{~h} /$ day, 7 days period) evoked the clearance of $A \beta$ soluble species and the decrease of $A \beta$ plaques in the auditory cortex and hippocampus of 5xFAD and APP/PS1 mice; both mouse models exhibit marked A $\beta$ plaque burden [30]. Similar results were found in another study [48] that used a longer stimulation protocol $(2 \mathrm{~h} /$ day, 15 days period) and reported that $40-\mathrm{Hz}$ AS stimulation decreased both soluble and insoluble $A \beta$ species, accompanied by a reduction in $\mathrm{A} \beta$ plaque number throughout the brains of $5 x F A D$ mice [48]. Interestingly, when AS was combined with another sensory modality (visual stimulation using light flickering at the same rate-40 Hz), a wider effect was produced across the brain, which exhibited reduced $A \beta$ pathology not only in the auditory cortex and hippocampus but also in the medial prefrontal cortex of 5xFAD mice [30].

In addition, only one study has reported a beneficial impact of AS on Tau pathology. By applying 40- $\mathrm{Hz}$ AS, Martorell and his collaborators (2019) observed a reduction in Tau hyperphosphorylation and seeding in the auditory cortex and hippocampus of P301S-Tau transgenic mice ( $1 \mathrm{~h} /$ day, 7 days period) [30]. No clinical evidence exists on the effectiveness of mechanical vibrations using any of the reviewed stimulation modes in mitigating Tau phosphorylation and accumulation in the $\mathrm{AD}$ human brain.

\section{Immunological response}

Among the reviewed studies performed in experimental models, 11 out of 14 have reported a positive immunological response induced by ultrasonic waves or AS. Again, no clinical evidence exists on the immunomodulation in the brains of $\mathrm{AD}$ patients or healthy, non-demented individuals under the three types of stimulation reviewed here.

TUSS The reviewed studies present conflicting outcomes concerning the effect of TUSS on the activity of glial cells (i.e., microglia, astrocytes, or macrophages) in the brains of AD transgenic mice. Most of the preclinical TUSS studies have reported activation of immune cells after treatment and their increased co-localization/internalization of $A \beta[36,56-61]$ or Tau [45] fragments in the brains of AD transgenic models. It is worthy to note that, despite the microglial activation (e.g., extended branching) after ultrasonic treatment, Leinenga and Gotz (2015) observed no alterations in inflammatory markers in the brains of aged APP23 mice (which exhibit pronounced A $\beta$ plaques) after US (once per week, 4 or 7 weeks period) [36]. On the other hand, two studies have reported a reduction in gliosis ( by assessment of glial fibrillary acidic protein [GFAP] [56] and ionized calcium-binding adaptor molecule 1 [Iba1] [56, 57]) in the brains of AD mouse models, either after a single TUSS session of $1 \mathrm{~min}$ (marked effect in the hippocampus and entorhinal cortex of $5 \mathrm{xFAD}$ mice) [56], or after a repetitive TUSS treatment (3 sessions/20 min each on alternate days, the first week of each month for three months) [57]. Note that the latter study used a significantly higher pulsing rate $(6 \mathrm{kHz})$ and longer treatment duration compared to the others and found a unique regulation of some immune-related genes (e.g., GFAP, Olig2, and CXC chemokine receptor 4) in the brains of 5xFAD mice after US treatment [57].

Moreover, Jordão et al. (2013) have demonstrated that TUSS (single session of $8 \mathrm{~min}$ ) increases the bioavailability of endogenous antibodies (i.e. immunoglobulins $G$ and $M$ ) in the brains of $A \beta$-based transgenic mice (TgCRND8), and also promotes the interaction of these antibodies with $A \beta$ plaques, thereby facilitating the clearance of their aggregates from the brain [59]. The increased antibody bioavailability is also accompanied by a phagocytic response of glial cells towards $A \beta$ aggregates in both US-treated and non-treated brain hemispheres [59], which is in line with previous reports supporting a US-induced systemic bioeffect in brain areas beyond the one that was stimulated [45]. Moreover, Lee and colleagues (2020) found a marked increase of $A \beta$ peptides in the cerebrospinal fluid space in the TUSS-treated 5xFAD mice, in which ligation of lymphatics to the deep cervical lymph nodes was performed (single session of $1 \mathrm{~min}$ ) [56]. The data from this study suggest that, although the reactivity of glial cells has been reduced by TUSS, A $\beta$ clearance could be ensured by the lymphatic system, which is possibly activated/stimulated by TUSS.

AS The $40-\mathrm{Hz}$ AS has been shown to modulate the immune response in the brains of $\mathrm{AD}$ transgenic mice with intense $A \beta$ plaque burden, by increasing microglial activity [30, 48] and astrocyte reactivity [30] (1 h/day, 7 days period [30]; $2 \mathrm{~h} /$ day, 14 days period [48]). However, the one-week-long AS study observed that the increase in microglial activation induced by AS was reversed one week after the end of the treatment [30]. Both studies have reported a decrease in soluble and insoluble $A \beta$ species $[30,48]$, possibly resulting from microglial activation. As the expression of inflammatory markers was not assessed, it was unknown if this increase in glial activation prompted or regulated neuroinflammation response. Importantly, combination of AS with visual stimulation delivered at $40 \mathrm{~Hz}(1 \mathrm{~h} /$ day, 7 days period) induced microglia to dis- 
play a unique encapsulating effect around $A \beta$ plaques throughout the brain of 5xFAD mouse, and this effect was not observed for any stimulus alone [30]. Importantly, this microglial activation results in decreased $\mathrm{A} \beta$ deposits in the brains of AD transgenic mice [30].

\section{Neurotoxicity and neuroplasticity deficits}

This section summarizes and discusses the effect of mechanical stimulation on different parameters of neuronal malfunction and neurodegeneration in $A D$, such as neuronal loss, synaptic malfunction, deficits in neurostructural plasticity (e.g., dendritic atrophy, spine loss), among others. Unfortunately, there is a lack of evidence for WBV or AS from human or animal studies. Therefore, this section only includes animal studies on TUSS.

TUSS treatment of various durations is associated with different beneficial effects against $A \beta$ - and Tauinduced toxicity and structural damage in the brains of AD transgenic mouse models. For instance, Shen (2019) and Lee (2020) led two experiments in which $1-\mathrm{Hz}$ ultrasonic pulses (single session of $1 \mathrm{~min}$ ) were applied to $3 \mathrm{xTg}-\mathrm{AD}$ and $5 \mathrm{xFAD}$ mice, respectively, which display advanced $A \beta$ and Tau neuropathologies $[56,58]$. The authors showed that the ultrasonic waves can modulate the proteomic profile in the brains of $\mathrm{AD}$ transgenic mice, which was correlated with enhanced neuronal function [58] and decreased neuronal loss [56] triggered by TUSS. In a study with longer TUSS stimulation, Burgess et al. (2014) found several morphological improvements (i.e., increased number of immature neurons, total dendrite length, and dendrite branching in pre-existing/ mature neurons) in the hippocampus of TgCRND8 mice (8 min once a week, 3 weeks period) [38], supporting that the beneficial effect of TUSS was also affecting different parameters of brain plasticity.

Also, Karakatsani et al. (2019) found that the brain slices from treated experimental animals show intact neural network integrity and neuronal morphology after unilateral target TUSS (single session of $1 \mathrm{~min}$ ), indicating treatment safety [45]. Additionally, Pandit and others (2019) have found TUSS-evoked increased autophagy in neurons (but not in glial cells) in Tau-transgenic K3 mice (2.4 min once a week, 15 weeks period) [66], showing a US-driven improvement of neuronal proteostasis and increased Tau degradation after an extended treatment period. Moreover, Eguchi and colleagues (2018) showed that TUSS in 5xFAD mice (60 min once a week, 12 weeks period) leads to the upregulation of endothelial nitric oxide synthase, neurotrophins, and heat-shock protein 90, accompanied by the downregulation of amyloid precursor protein (APP) and beta secretase-1 (BACE-1), which are associated with a reduction of $A \beta$ species and plaques throughout the brain after TUSS
[57]. Importantly, Pandit et al. (2019) have also reported reduced p-Tau levels and neurofibrillary tangles in the hippocampus of an AD mouse model, possibly related with the increased autophagy [66]. Again, no clinical evidence has been provided on the mitigation of neurotoxicity after US treatment in humans.

\section{Brainwave entrainment and brain functional connectivity}

$W B V$ Starting with this therapeutic modality, only one of the reviewed papers has assessed the impact of an eightweek WBV program on neuronal activity of the human brain [67]. Specifically, this study reported increased brain network activity (assessed by electroencephalography, EEG) overtime after $20-\mathrm{Hz}$ to $40-\mathrm{Hz}$ vertical vibration (frequency increased over treatment time) in women with senile dementia [67].

TUSS US treatment has also been proven to increase neuronal activity, despite the limited experimental evidence on the topic. Indeed, among the reviewed studies, only one animal study has reported the occurrence of strong and transient entrainment of $40-\mathrm{Hz}$ oscillations in the brains of 5xFAD mice during TUSS pulsing at the same frequency (single session of $1 \mathrm{~h}$ ) [61]. At the clinical level, two studies have reported alterations in brain networks after US therapy in AD patients. Meng and colleagues (2019) observed a decreased resting-state functional connectivity in the right frontoparietal networks during $\mathrm{BBB}$ opening, although the functional organization was restored at the one-day and one-week followups (2 US sessions, one-month apart) [68]. In the same study, the US-treated patients showed a small tendency of increase of brain connectivity at the 3-month follow-up, both in the right frontoparietal and in the default mode networks [68]. In fact, Beisteiner et al. (2020) have studied the effect of 6-12 sessions delivered within the same time window - 1 month period - and detected enhanced memory networks, as well as increased steady-state and task-based functional connectivity in the hippocampus, cortex regions, and the precuneus, and in bilateral hippocampi, respectively [69]. These findings suggest that transcranial US application could be effective in modulating brain activity and networks possibly by adopting frequent sessions over a longer period. In addition, the brain network modulation is correlated with cognitive improvements in $\mathrm{AD}$ patients, after both two- or four-week interventions [69]. Thus, it is quite plausible that the periodicity of stimulation can greatly affect the effectiveness and durability of the treatment.

AS Five out of the six reviewed studies assessing the effect of AS (two preclinical and three clinical studies) have shown that AS is effective in increasing neuronal 
activity [30, 31, 48, 49, 70]. The two preclinical AS studies observed increased gamma power after delivery of $40-\mathrm{Hz}$ intermittent sound waves in 3-8 month-old wildtype (C57BL/6 J) mice ( $1 \mathrm{~h} /$ day, 7 days period) [30], and in 5xFAD transgenic mice ( $2 \mathrm{~h} /$ day, 14 days period) [48]. Interestingly, evoked gamma brain oscillations promoted microglia aggregation in both transgenic mice and their wild-type littermates [48], suggesting that this immunomodulation response is not necessarily activated in response to $A \beta$ and/or Tau pathologies. This brainwave entrainment was accompanied by increased functional connectivity in frontal gamma coupling with parietal delta at the baseline, which swapped to frontal gamma coupling with parietal theta after TUSS [48]. However, an exaggerated increase of evoked gamma brainwaves was observed one day after the intervention, which returned to baseline levels during the rest of the experiment [48], indicating an acute and transient response. In addition, Martorell et al. (2019) have shown that $40-\mathrm{Hz}$ visual stimulation combined with AS delivered at the same frequency $(1 \mathrm{~h} /$ day, 7 days period $)$ shows a greater gamma oscillation entrainment followed by decreased $A \beta$ load in the brains of $5 x F A D$ mice when compared to each of the stimuli alone.

In line with these findings, the first, and only so far, clinical evidence on the improvement of brain network activity after AS combined with visual stimulation in $\mathrm{AD}$ patients is provided by Calomeni and colleagues (2017) (15 $\mathrm{min} /$ day on alternate days, 20 days period) [49]. This study found that these sensory stimuli delivered at $8,10,12,14$, and $15 \mathrm{~Hz}$ (3 min/frequency) increase alpha (i.e. $8-15 \mathrm{~Hz}$ ) brainwave activity in $\mathrm{AD}$ patients, but not in non-demented individuals [49], which is associated with memory gain [49]. Moreover, Papalambros and colleagues performed two experiments in 2017 and 2019, in which they assessed the effect of $20-\mathrm{Hz}$ AS (single session of 4 min delivered during sleep) on the neural activity in healthy non-demented older subjects [70] and patients with amnestic $\mathrm{MCI}$, a precursor form of $\mathrm{AD}$ [71]. In the first study performed in healthy non-demented older subjects [70], a unique increase of slow-wave brain activity in the delta range (i.e. $0.5-4 \mathrm{~Hz}$ ) during stimulation is detected, accompanied by a small tendency of enhanced theta and fast spindle power. Moreover, increased signal amplitude and spindle density are observed during stimulation for all potentials, as assessed by EEG [70]. Importantly, the increased slow-wave activity (i.e. $0.5-4 \mathrm{~Hz}$ ) is associated with better performance in word recall after stimulation [70]. By applying the same AS protocol to amnestic MCI patients, the authors have found increased slowoscillations (i.e. $0.5-1 \mathrm{~Hz}$ ), slow-wave and sigma (i.e.
9-15 Hz) activities during stimulation, whereas no effect was noticed in theta $(4-7 \mathrm{~Hz})$ or beta $(16-20 \mathrm{~Hz})$ bands [31]. Again, the alterations in neural activity are correlated with improved memory and cognition, as assessed by the cued-recall and verbal paired-associate tests, as well as by the NIH Toolbox Cognition Battery [31].

\section{Behavioral performance}

The reviewed studies typically present outcomes associated with motor and behavioral abilities. Twenty-seven out of the 37 analyzed papers provided evidence on the effect of mechanical vibration-based stimulation on motor and cognitive performance.

\section{WBV}

Despite the differences among the protocols (vibrations at $25 \pm 15 \mathrm{~Hz}, 5-\mathrm{mm}$ maximum amplitude, and heterogeneous sessions and treatment durations), studies have shown that the WBV treatment can improve different aspects of physical function, such as balance [21, 72-76], functional mobility [21, 72-79] and muscle mass and/or strength, mainly in the lower limbs [72, 76-78]. Importantly, three studies have reported an enhancement of quality-of-life metrics after WBV training (6-9 weeks period) $[21,73,78]$, which are directly associated with motor improvement, as well as with patients' well-being [80]. In addition, Kim and Lee (2018) showed that WBV could improve cognitive function along with increased brain connectivity and plasticity in senile-demented women undergoing a WBV regimen with increasing frequency $(20-40 \mathrm{~Hz})$ of vibrations over time (4 min once a week, 2 months period) [67]. However, the beneficial effect of WBV treatment in non-demented older adults remains unclear, as a WBV treatment of 10 weeks (1-5 min twice a week, 10 weeks period, with increasing training volume over time) described a small tendency (but not significant effect) of functional improvement as assessed by different motor ability tests like walking speed metrics [81]. Future studies should clarify whether the WVB treatment is also beneficial for non-demented older individuals.

TUSS TUSS is also associated with improvement of motor and cognitive skills in AD animal models, including enhanced different types of memory (i.e. recognition, spatial, working, and short- and long-term memories) $[36,56,58,66]$, improved exploratory skills [36, 38, 57] and improved motor ability and coordination [66]. Interestingly, after weekly sessions of 2.4-min delivery over 15 weeks (the longest of the reviewed protocols), no changes in grip strength (a motor-related parameter) were found in $\mathrm{K} 3$ mice. [66]. In addition, a study in aged 
$A \beta$-positive dogs with advanced $A \beta$ brain pathology found no significant changes in gait, postural reaction or cranial nerve testing after TUSS (2-3 $\mathrm{min} / \mathrm{session}$, once a week, 4 weeks period) [62].

At the clinical level, Lipsman et al. (2018) did not find significant changes in cognition and daily functioning of AD patients at 3-month follow-up after TUSS (7.5 min once a week, 3 months period) [63]. However, in the same study, one of the five treated patients showed a transient cognitive improvement at 1-month follow-up [63]. Similarly, other studies have detected no significant changes in cognitive function in $\mathrm{AD}$ patients after two to five sessions of continuous ultrasonic waves $(220-\mathrm{kHz}$ center frequency) (2 weeks period), either at 30-day [82] or at 1-week follow-up [65], independent of alterations in $A \beta$ burden. On the other hand, one study has demonstrated that a US treatment delivered in $5-\mathrm{Hz}$ ultrasonic pulses ( 2 to 4 weeks period) is able to improve cognitive performance (e.g. enhanced memory and verbal processing) in a standardized neuropathological assessment defined at the Consortium to Establish a Registry for Alzheimer's disease (CERAD), in a cohort of AD patients right after the treatment as well as three months later [69]. Importantly, the cognitive improvement of these $\mathrm{AD}$ patients is correlated with increased functional connectivity between different brain areas (e.g. hippocampus, parahippocampal cortex, parietal cortex) [69].

$A S$ For AS, a majority of evidence for its beneficial impact on behavioral performance has arisen from clinical rather than animal studies. Only the animal study by Martorell et al. (2019) reported improved spatial memory and recognition in 5x-FAD mice after $40-\mathrm{Hz}$ AS $(1 \mathrm{~h} /$ day, 7 days period), as assessed by different behavioral tests (i.e., Morris water maze, novel object recognition, and novel object location tasks) [30]. In human studies, multiple insights on behavioral performance and cognitive function have been recently published. For instance, Papalambros et al. assessed the impact of a $20-\mathrm{Hz}$ AS intervention (single session of 4 min during sleep) in nondemented elderly [70] and amnestic MCI patients [31] and found a positive effect on morning word-recall in MCI patients [31].

Finally, vibroacoustic stimulation (type of AS-) combined with visual stimuli, both delivered at $40 \mathrm{~Hz}(30 \mathrm{~min}$ twice a week, 6 weeks period), improves cognitive performance after stimulation in AD patients, as well as the awareness of surroundings, the interaction and discussion/storytelling abilities, and alertness [83]. Overall, the data suggest that $40-\mathrm{Hz}$ stimulation delivered in different rhythmic sensory-based modalities is a promising tool to improve different aspects of behavior.

\section{Discussion}

The ability of noninvasive, mechanical vibration strategies to impact $\mathrm{AD}$ pathology and related behavioral deficits has received increasing attention over the last years. Hereby, we provide a systematic review comparing and discussing cellular, molecular, functional, and behavioral outputs of three mechanical-based stimulation modalities and the most promising protocols against the $\mathrm{AD}$ (Fig. 2).

\section{Safety and feasibility of mechanical vibration-based interventions}

For the clinical implementation of any therapeutic modality, it is imperative to assess the safety and feasibility of the treatment. Particularly, WBV training requires a wise consideration of such parameters, especially in older individuals. These features have been extensively considered in the reviewed studies, as many of them are important for the participation of the elderly in the implemented programs, including the attendance rate, compliance, feedback provided by the participants, and adverse events. All the reviewed WBV protocols present a high attendance rate $(>74 \%)$ and treatment compliance $(>73 \%)$, and scarce adverse effects (e.g., muscle pain and discomfort) are reported after vibratory stimulation. The implemented protocols are well-tolerated by the majority of the participants. For instance, while a $30-\mathrm{Hz}$ WBV training protocol in demented patients is reported as enjoyable by the participants [84], another study of $26-\mathrm{Hz}$ vibrations reported that the subjects felt high discomfort, forcing the protocol to be adapted to a vibration frequency of $18 \mathrm{~Hz}$; yet, the 18- $\mathrm{Hz}$ protocol was also poorly tolerated by the elderly [81]. These data suggest that the discomfort is not frequency-dependent, but possibly caused by a greater amplitude of vibration. Indeed, WBV protocols with vibration rates over $30 \mathrm{~Hz}$ have proved safe and well-tolerated by the elderly among the reviewed studies [21, 77, 79, 84]. In contrast, high-amplitude vibration should be avoided to prevent distress and dropouts from the WBV training. Importantly, the reviewed studies suggest that WBV protocols are suitable even for frail patients $[76,78,81]$ and individuals with cognitive impairments [21, 67, 84], as they do not require any physical effort or ability and can also be personalized for each patient according to his anatomical and physiological features. Taken together, these data demonstrate that WBV should be added to medical and rehabilitation therapies in older patients, enabling the enhancement of their neuromuscular performance and, possibly, cognitive skills.

Regarding the US modalities, the US-mediated BBB opening has proved safe, transient, and effective in all the reviewed protocols. No major adverse events occur and TUSS is well tolerated by all animals and patients. 
However, among the studies reviewed, no long-term effects were assessed, with the longest follow-up period in AD patients being three months [69]. Nevertheless, a study in healthy non-human primates employing repeated ultrasonic waves over four to 20 months has reported no damage in the animal brain, and no alterations in health status (e.g., respiration rate, blood pressure, and $\mathrm{O}_{2}$ level) throughout the intervention [85]. These findings support the long-term safety of US treatment in primates, but this issue needs to be addressed in future transcranial US studies in both demented and non-demented patients, mainly after multiple-sonication protocols. Importantly, to prevent tissue damage and ensure appropriate sonication power, some of the reviewed studies [38, 63, 68] used an acoustic feedback controller algorithm proposed by O'Reilly and Hynynen (2012) [86] that allows the real-time monitoring of acoustic emissions. The system detects subharmonic emissions that indicate enhanced microbubble activity, and responds by reducing the applied acoustic pressure in the stimulation site. This enables the control of ultrasound emission according to microbubble response, avoiding in situ pressure fluctuations due to skull thickness or vasculature variations among individuals [38]. This represents an important development in the field in terms of clinical translation of TUSS for AD, contributing to good tolerability and safety.

Finally, sparse information exists on the safety and feasibility of AS protocols since this sensory modality does not require any effort, or induce any pain or discomfort in participants. In fact, when applied overnight, both healthy non-demented older adults and amnestic MCI patients report no changes in sleep quality and sleepstaging features [31, 70], suggesting that AS is comfortable and suitable even for demented elderly persons.

\section{Most relevant outcomes of multi-mechanical stimulation}

Based on the reviewed studies (Table 5), we found that the main outcomes associated with the WBV program in the elderly are improvement of different aspects of motor performance, such as mobility and balance skills, as well as increased muscle mass and/or strength. The majority of the reviewed studies have reported enhancement of at least one of these parameters, although a wide variety of WBV protocols have been implemented. Intriguingly, we noticed some divergent outcomes even for similar intervention parameters. We assume that this divergency is associated with differences in the patient's physical/ motor status at baseline. As suggested by Lam (2017), worse baseline physical/motor status allows for a greater gain of physical and/or cognitive performance after WBV treatment [21], which can explain the lack of significant results in some studies due to insufficient mobility impairment, and so, less improvement. Although this approach seems to be more efficient in people with worse neuromuscular function, this does not mean that nonfrail subjects cannot benefit from WBV training. In fact, many studies have reported marked improvement of motor and cognitive performances in young and healthy adults [87-90]. Future studies in older individuals should address the baseline functional mobility and cognitive status to understand the suggested reversed relationship between improvement and health status.

Nevertheless, it has been demonstrated that the implementation of WBV training programs in demented and cognitively impaired individuals is effective in improving the overall neuromuscular function, functional mobility, and metrics related to the quality of life $[21,73,78]$. Simultaneously, this training may also improve disrupted brain networks and many aspects of cognitive function, such as orientation, memory, and linguistic skills [67]. Importantly, by enhancing the overall motor ability, WBV exercise may constitute an effective strategy to prevent falls and therefore bone fractures [76, 91], as well as brain damage due to trauma, which are common in advanced age [92, 93]. Furthermore, the improved neuronal activity and cognitive enhancement induced by WBV in seniledemented women [67] point towards further investigation on the effect of WBV on cognitive status and brain pathology in $\mathrm{AD}$ or demented patients.

On the other hand, TUSS can provide noninvasive immunoregulation and neuromodulation reversing $A D$ pathomechanisms. Glial cells play a critical role in maintaining the balance in the nervous system, mainly when something disrupts its functioning. For instance, in the AD brain, microglia and astrocytes play an essential role in controlling the levels of aggregated proteins (mainly $A \beta$ ), promoting their clearance. For this reason, many researchers have focused on immunotherapies to increased $A \beta$ clearance through administration of immunotherapeutic agents, both related and non-related with microglial activation [94], although non-satisfactory results have been obtained in clinical trials [95]. Here, many of the TUSS studies have shown activation of different immune cells, enhanced co-localization with $A \beta$ and Tau followed by reduced $A \beta$ or Tau accumulation in the brains of different AD transgenic mouse models [38, 66]. In fact, several researchers have reported enhanced co-localization of microglia around $A \beta$ or Tau aggregates in the brains of AD transgenic mice after US stimulation $[36,45,56-61]$ as well as $40-\mathrm{Hz}$ AS combined with visual stimulus in 5xFAD mice [30]. Importantly, these alterations are accompanied by a reduction of $A \beta$ or Tau deposits [45, 56-59, 61]. Stimulated by Iaccarino et al. (2016) who used $40-\mathrm{Hz}$ visual stimulation to produce gamma brainwave entrainment in the brains of $\mathrm{AD}$ 
transgenic mice, many research efforts have provided strong evidence about the effectiveness of TUSS pulsing at low frequency. For instance, $5-\mathrm{Hz}$ and $40-\mathrm{Hz}$ USS modulate brain network activity in AD transgenic mice [61] and patients [69], respectively. Moreover, pulsed TUSS evoked neurogenesis and dendritic growth in the hippocampus of TgCRND8 mice [38,57]. This evidence supports the growing interest in ultrasonic wave-based treatments to fight against AD brain pathology, not only by blocking or reversing $A \beta$ and/or Tau pathology but also by attenuating inflammation, neuronal and synaptic loss, and altered brain activity.

As AD patients present abnormalities in gamma oscillations that are essential for high-order cognitive functions, such as information storage and retrieval [54], these brainwaves have been used as a target in emerging strategies to address altered brain networks in the AD brain. The sensory-based modalities delivered as rhythmic stimuli, which particularly focus on the gamma frequency band, have gained major attention in the last five years to improve altered brain oscillations; increased brainwave power and synchronization seem to affect $A \beta$ and/or Tau pathologies [30, 48, 51, 54]. In fact, the association between brainwave entrainment and AD neuropathology has been widely studied, as researchers have found that the entrained brain oscillations can modulate AD neuropathology, reducing $A \beta$ and/or Tau pathology $[51,54,96]$. Moreover, other frequency bands have also been associated with memory and cognitive enhancements. For instance, visual and auditory stimuli delivered at alpha and beta frequency bands can increase brain oscillations in EEG signals associated with memory enhancement [49]. Interestingly, these stimuli affect brainwave activity differently according to the conditions of the participants, as the applied therapy increases alpha activity in the brains of AD patients while no significant effect was produced in patients with Parkinson's disease and non-demented older individuals [49]. This suggests that the applied protocol is more effective in improving neuronal network dysfunctions associated with $\mathrm{AD}$ neuropathology. Similarly, Papalambros et al. (2017 and 2019) observed increased slow-wave activity (i.e. $0.5-4 \mathrm{~Hz}$ ) in both non-demented and demented patients after $20-\mathrm{Hz}$ AS (beta frequency band) overnight, which is correlated with cognitive enhancement $[31,70]$. Interestingly, these studies show that the increased power of specific brainwaves is not necessarily associated with stimuli delivered in the same frequency band, suggesting that rhythmic AS can increase brain activity across different frequency bands.

\section{Most promising protocols for clinical application}

In WBV studies, the passive exercise training is delivered using two distinct vibration modes, namely verti$\mathrm{cal}$ and rotational vibration. These modes differ in terms of vibration mechanisms, the direction of vibration, and stimulated muscles, and hence they can produce different outcomes in similar cohorts. For this reason, it is important to clarify which aspects of these vibration modes can lead to beneficial or detrimental effects in the frail elderly. On the one hand, mechanical acceleration in rotational vibration is performed in the anterior-posterior axis [14], which is coincident with the direction in which the lower limb balance declines over the aging process; rotational vibration can also cause muscle and joint pain [97]. However, rotational vibration has been proven to promote an increase in muscle strength, which has been associated with the increased balance and functional mobility [77, 98]. On the other hand, in the WBV training with vertical vibration, the platform moves up and down, which mobilizes all body weight, toning muscles and strengthening bones [99]. As both types of vibration are associated with positive outcomes, further investigations should compare and assess the impact of different directions of the vibration in the elderly and particularly $\mathrm{AD}$ patients. In addition, many studies have used a WBV protocol with progressively increased frequency of vibration over time, which is associated with marked improvements in neuromuscular performance and/or quality of life [73, 77-79]. This suggests that increasing the training intensity during the WBV treatment may elicit more beneficial effects than using a constant vibration frequency. In summary, data extracted from the reviewed studies suggest that the main parameters to be employed in future AD clinical research on WBV protocols are: (i) vertical vibration, as only this type of vibration is used for cognitively impaired subjects; (ii) pulse frequency of $20-40 \mathrm{~Hz}$, with special emphasis on $30-\mathrm{Hz}$ vibration and increased frequency over time; (iii) amplitude of vibration of $2 \mathrm{~mm}$; and (iv) a training volume of 4-6 min per session, two to four sessions per week over five/six consecutive weeks. Importantly, although the treatment parameters among cohorts are quite similar, there is a tendency for shorter treatment (i.e., lower duration) employed in demented patients compared with non-demented subjects.

Considering TUSS in patients with AD or dementia, the effectiveness needs to be preceded by a demonstration of the intervention safety, mainly due to the potential thermal and damaging effects produced by the US when applied at a high frequency. For this reason, and given the information provided in Table 3, we believe that future protocols designed to affect $\mathrm{AD}$ pathology should consider: (i) the frequency of the pulse, as most of the reviewed preclinical studies used intermittent stimulation 
(commonly from 1 to $10 \mathrm{~Hz}[36,37,58,66,69]$, although $40-\mathrm{Hz}$ TUSS was shown to produce strong but transient signal entrainment in the brains of AD transgenic mice [61]); (ii) the delivery mode, as the whole-brain stimulation (e.g. through a helmet-like device) seems to be the most appropriate option to produce a widespread effect over the brain, although localized sonication has proved to affect further brain regions in mice $[45,56,59]$; and (iii) the number of sonications, as a minimum of six sessions are required.

Finally, concerning the application of AS in AD and cognitively impaired patients, the most important parameters for the effect of AS on AD pathology are: (i) pulsing rate of $40 \mathrm{~Hz}$, which represents the most promising option (essentially based on AD transgenic mice studies); and (ii) stimulation volume of 15 - to 30 -min sessions, at least once per week, over a minimum of three weeks. However, a single session of overnight AS during sleep is also effective in increasing slow-wave activity and enhancing memory recall in non-demented older adults [70] and in amnestic MCI [31], which means that AS delivery during sleep should be further investigated in AD patients in future trials.

Overall, the reviewed protocols show that various combinations of protocol specifications can be employed to improve different aspects of $\mathrm{AD}$ pathology and to improve cognition and other behavioral aspects. Currently, quite similar treatment parameters have been employed in both demented and non-demented subjects. In further studies in $\mathrm{AD}$ and demented patients, it is necessary to determine which protocol specifications should be employed to impact $\mathrm{AD}$ pathology.

\section{Limitations and future directions}

The main limitation associated with the reviewed studies is the lack of histological/biochemical and/or imaging assessment of key biomarkers, such as $A \beta$ and Tau species, and neuronal damage/inflammation markers in the brains of participants. This monitoring would enable further advances in the development of mechanical-based treatments to block AD pathomechanisms. Most of the studies performed in humans essentially focus on brain network activity and motor and behavioral improvements, while only three studies have monitored alterations at biochemical and functional/structural levels (e.g., serological analysis to assess biomarkers, and brain imaging techniques such as PET and MRI) after mechanical wave treatment $[63,65,100]$. Therefore, additional studies that use mechanical-based stimulation modalities and report biochemical and imaging brain data are needed to clearly describe the bioeffects of mechanical-based stimulation modalities on different molecular and metabolic aspects of $\mathrm{AD}$ neuropathology.

Moreover, longer follow-up periods are needed to test the effect of detraining or treatment suspension, as most of the reviewed studies with a follow-up period have reported a decline/reverse of the positive effect right after the treatment $[37,59,63,76]$. In fact, no clinical data have been published so far on the longterm effect of these stimulation modalities, so a longer study span is needed in the future.

In addition, many of the reviewed studies present a lack of important information related to protocol specifications, e.g. the amplitude of vibration $[67,75]$ and the type of vibration [84] in WBV protocols, as well as the US center frequency [69] and pulsing rate [63, 65, $82]$ in TUSS studies. Other parameters such as the contact area and pressure of transducer on the heads of animals/patients, the distance and angle between the transducer and the head, the duty cycle, and the intensity of acoustic signal (e.g., power density) are required to fully characterize the mechanical stimulation protocol, which allows for reproducibility in experiments and a reliable comparison among different studies.

\section{Conclusions}

Latest studies have reported the effectiveness, safety, and feasibility of mechanical waves delivered through different stimulation modes to mitigate $\mathrm{AD}$ pathology and to improve the overall physical and behavioral performance of AD patients, demented individuals, and non-demented elderly persons. Importantly, clinical evidence from $\mathrm{AD}$ and demented patients has accumulated over the past five years, and promising results have been generated. Based on the current evidence, the low-cost and noninvasive mechanical-based interventions can contribute to the development of an alternative effective solution to fight AD neuropathology and related behavioral deficits. However, there is limited evidence on the comparison between treatment specifications, and hence, suboptimal and quite distinct protocols have been employed. This comparison is crucial to further optimize the stimulation protocols in order to maximize their therapeutic potential. Nevertheless, the effectiveness of the mechanicalbased stimulation strategies in promoting physiological and electrochemical alterations that could mitigate $\mathrm{AD}$ pathology will certainly evolve in the future and, hopefully, revolutionize $\mathrm{AD}$ therapeutics in the upcoming decades. 


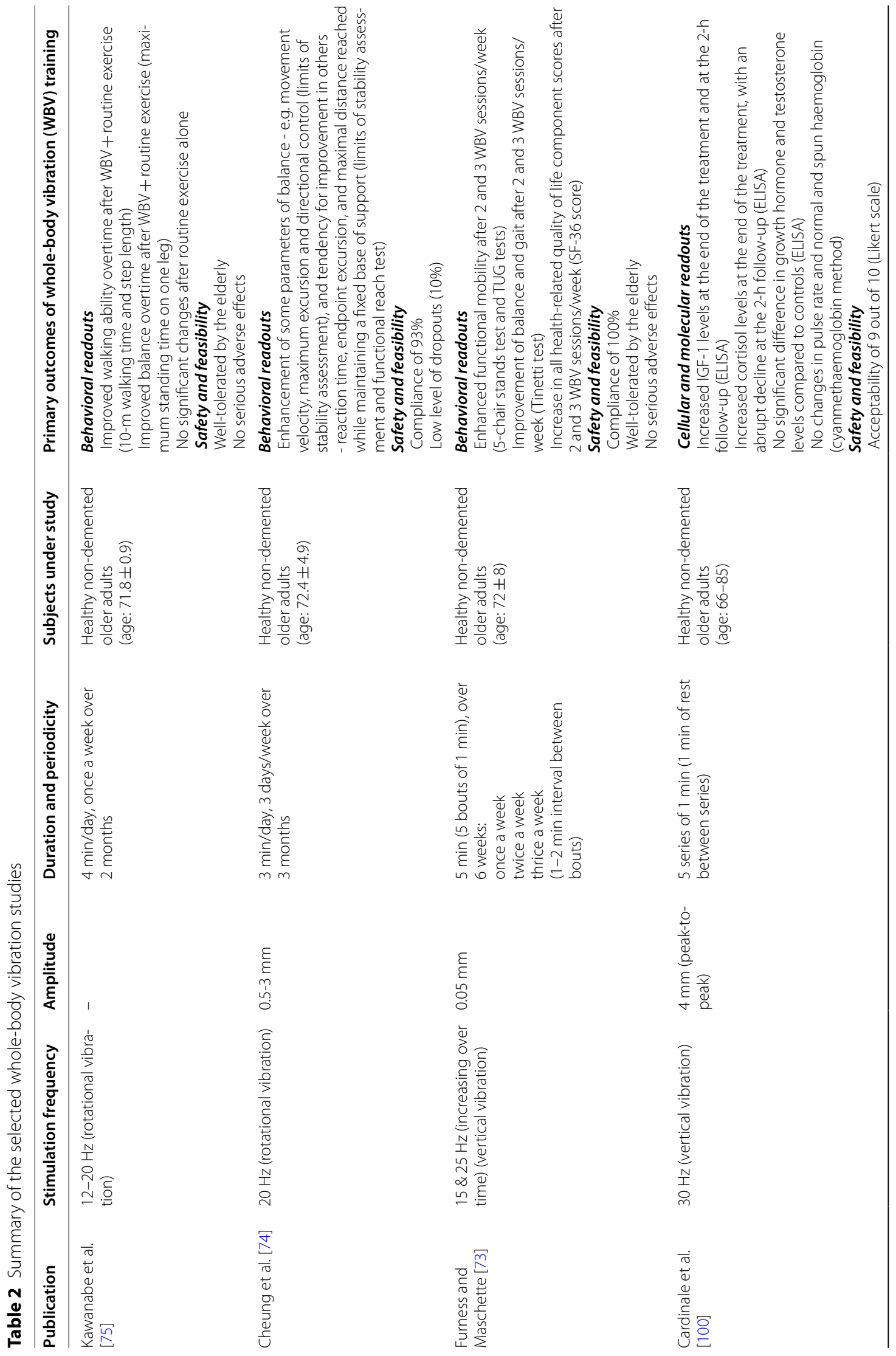




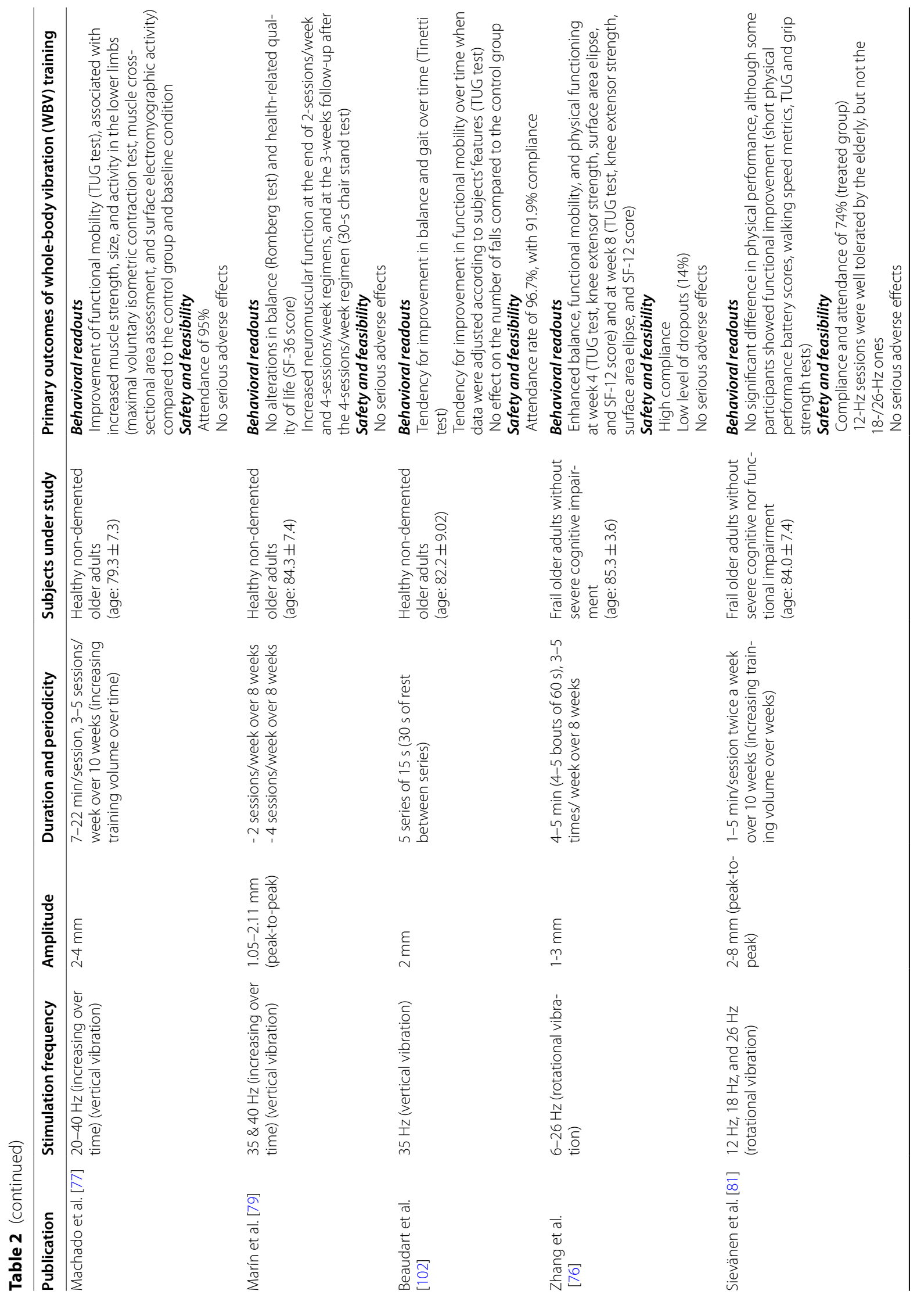




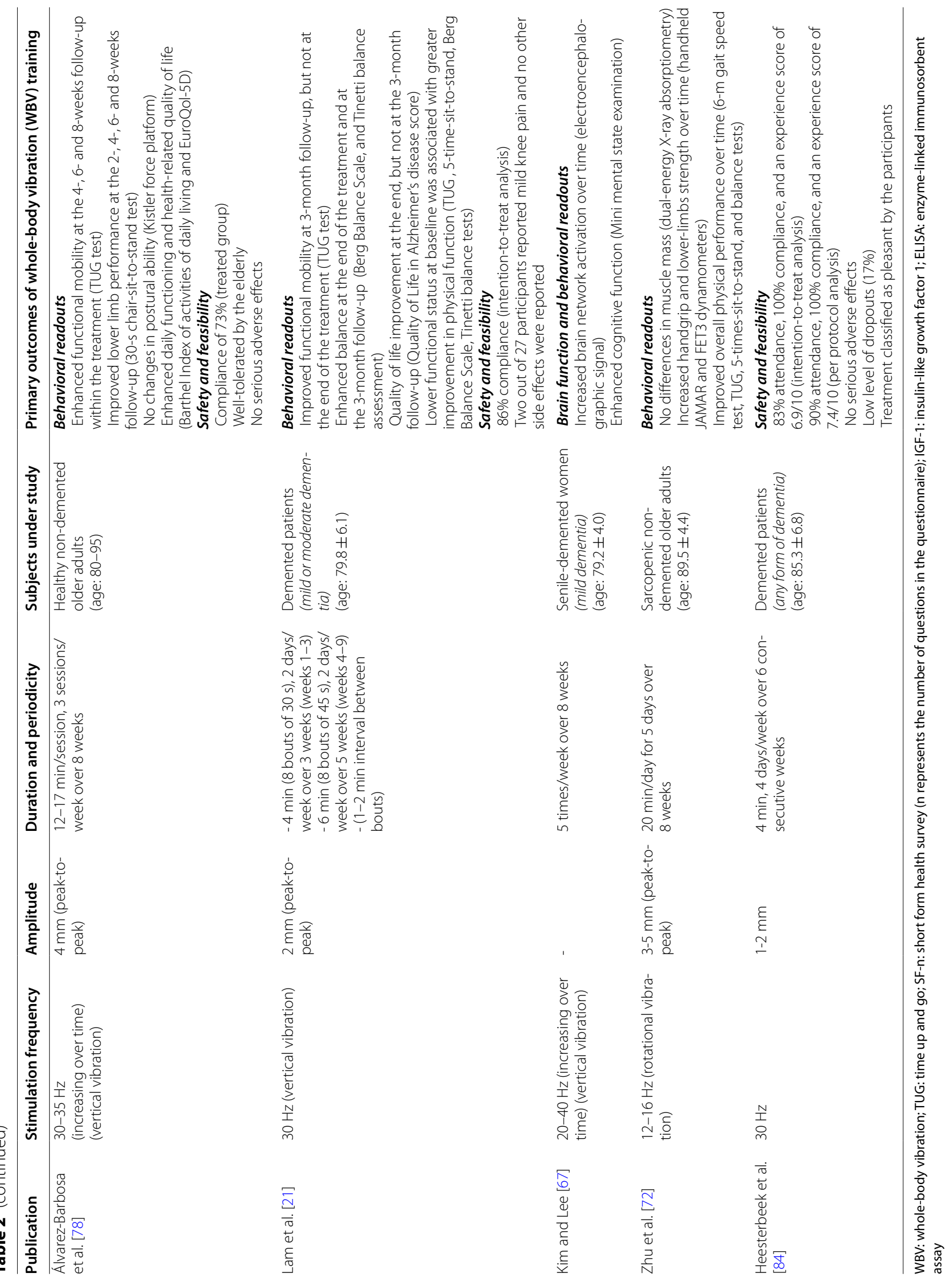




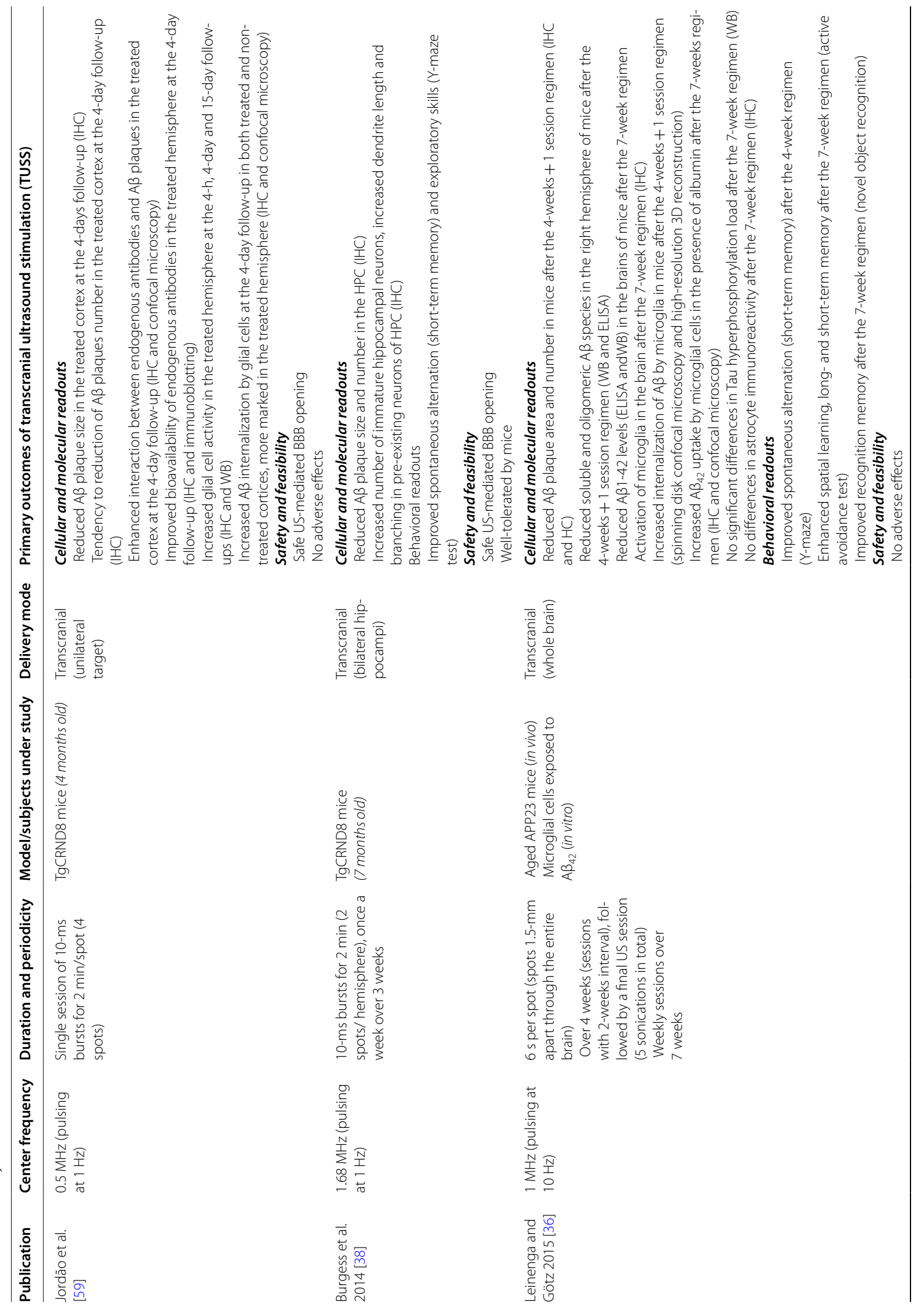




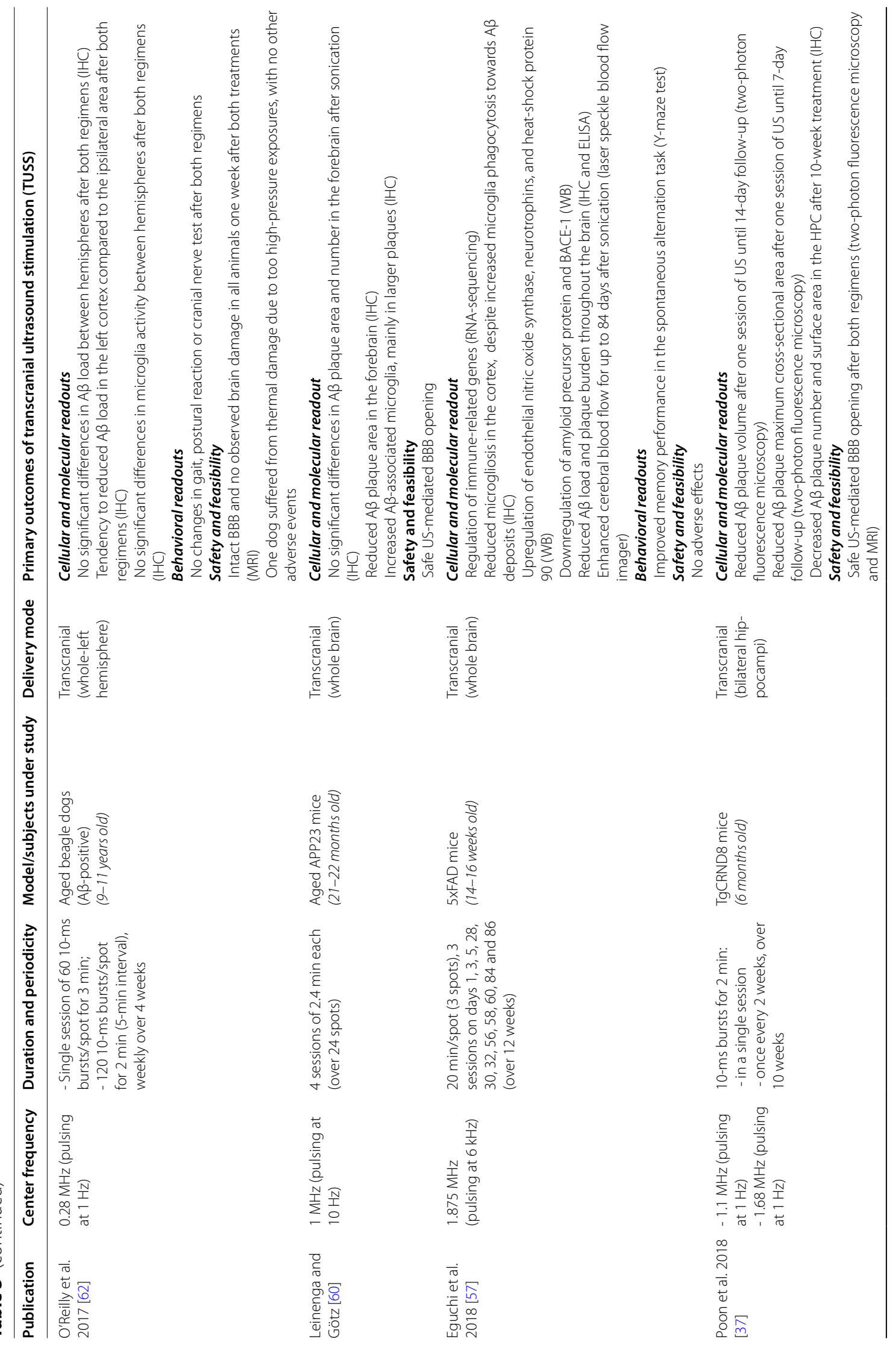




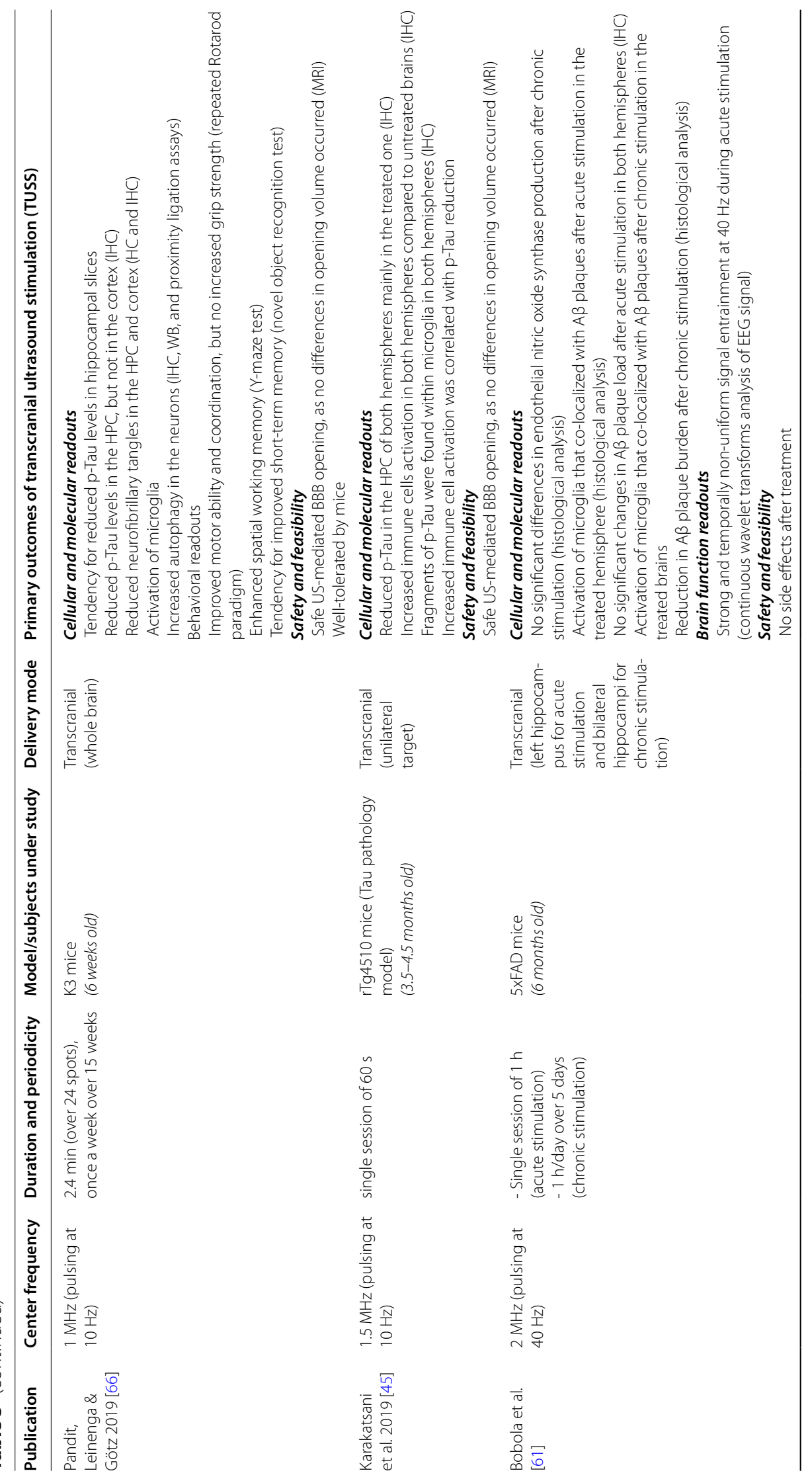




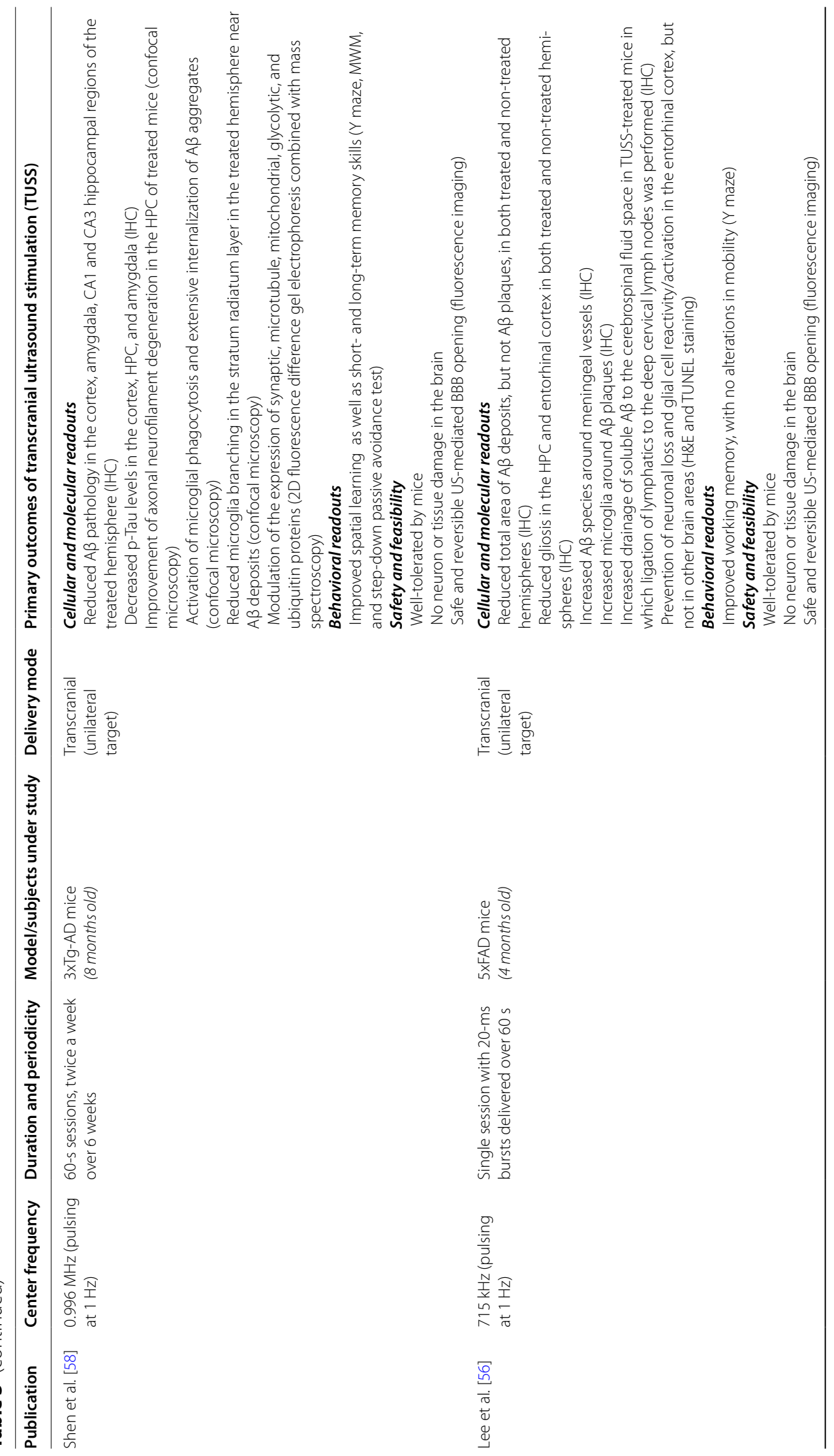




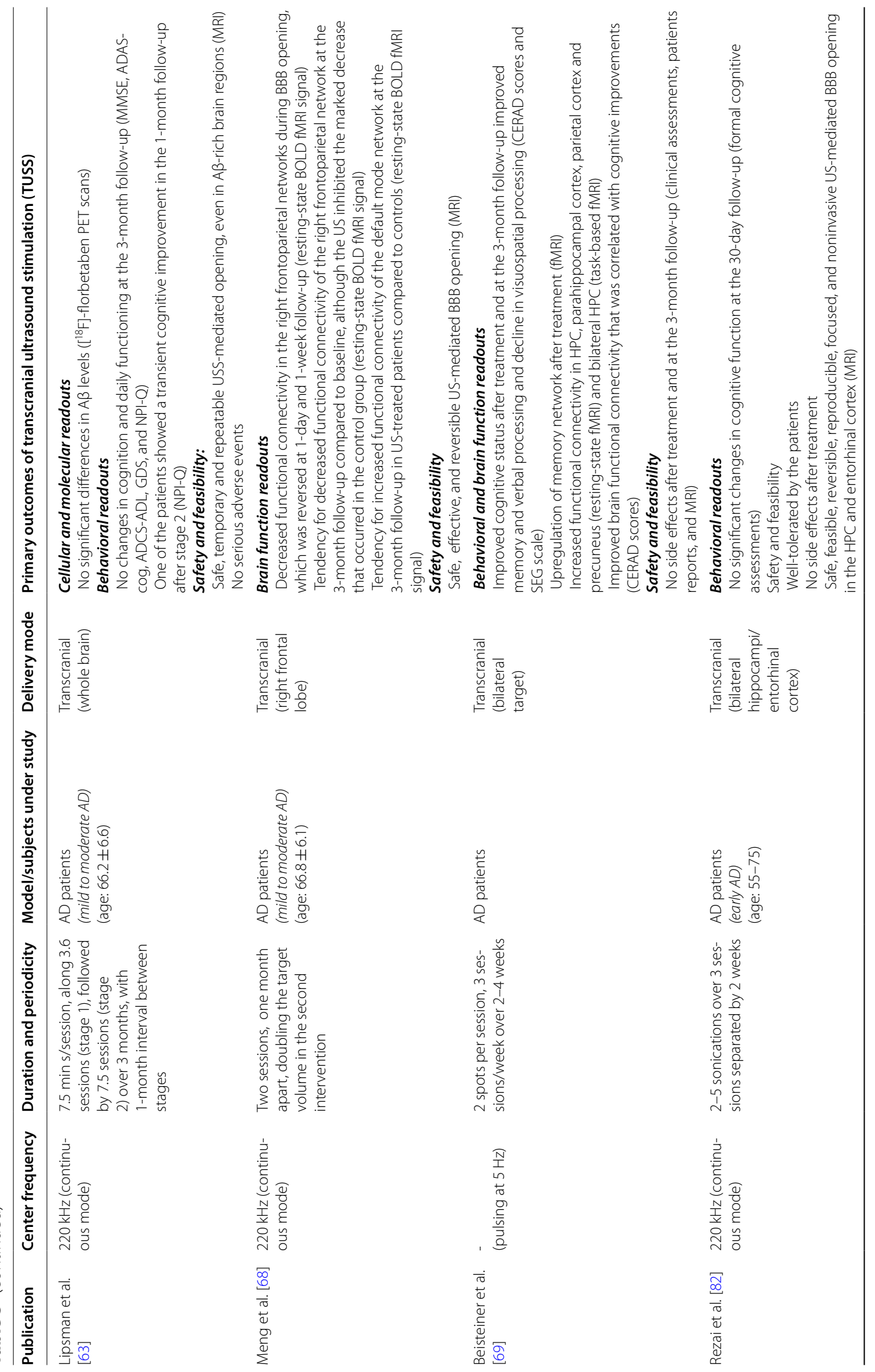




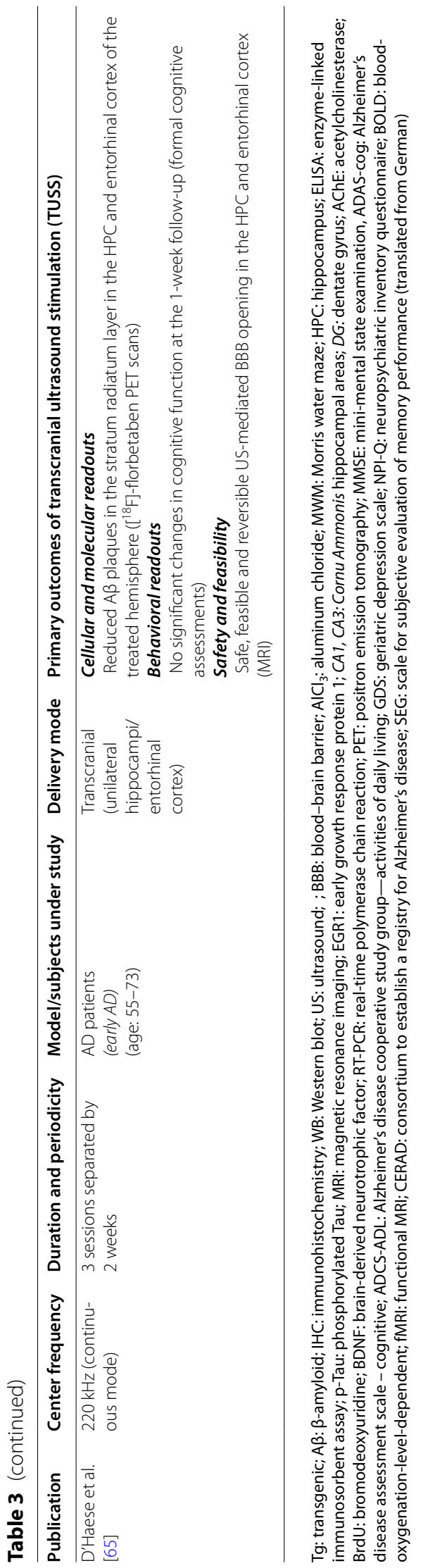




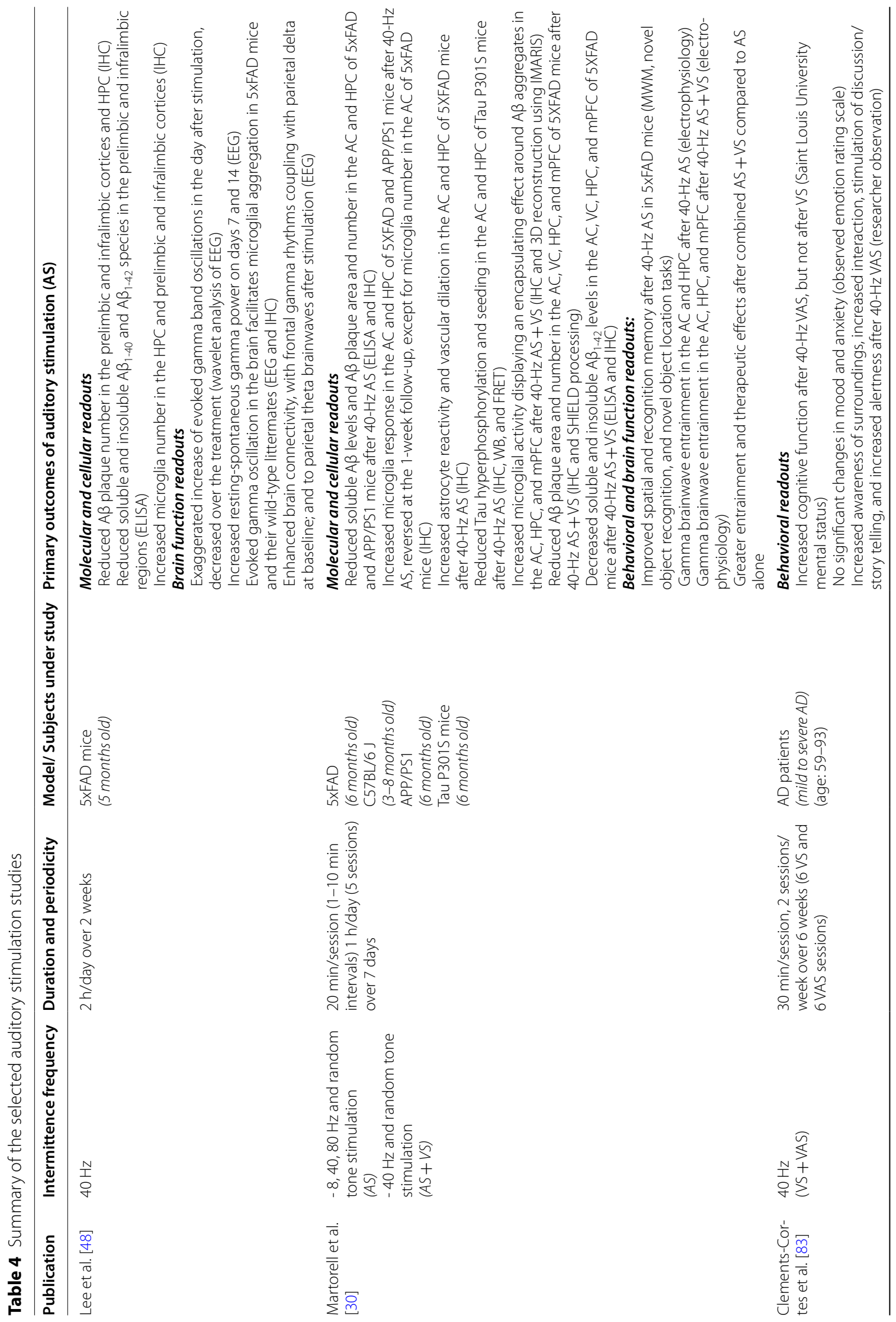




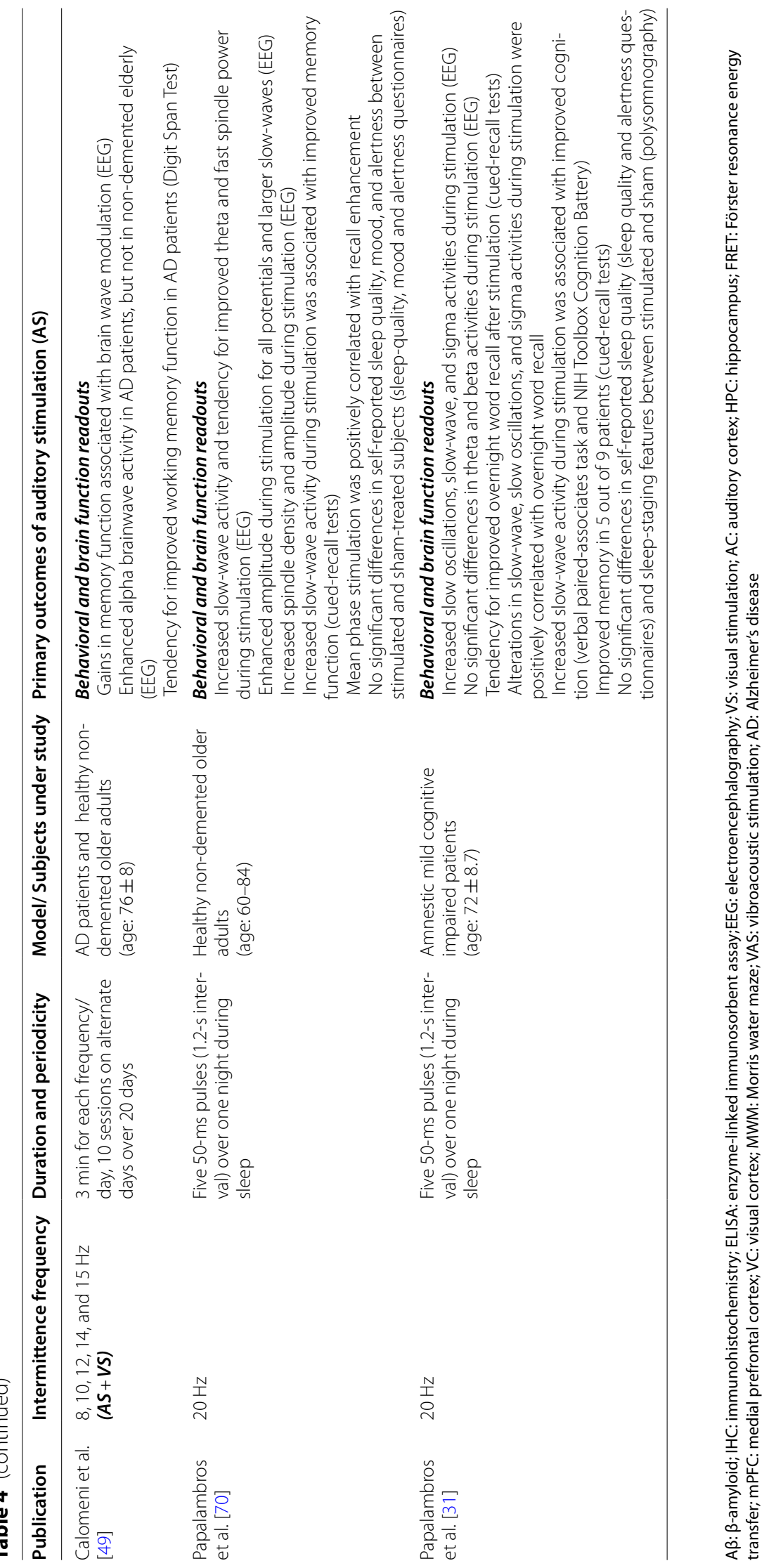


Table 5 List of the aspects of AD pathology in which mechanical-based modality produced positive effects for each of the reviewed studies

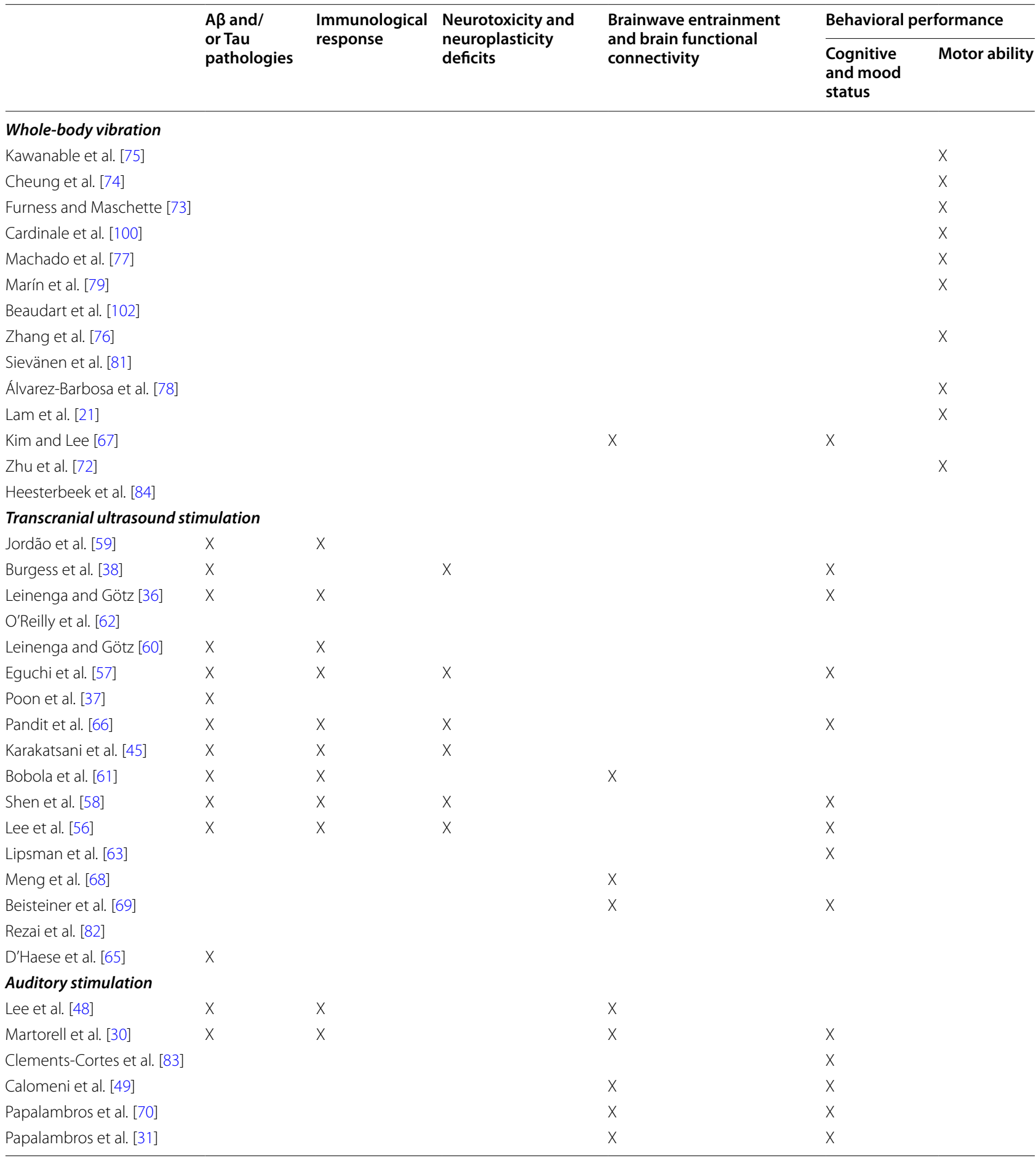




\section{Whole-Body Vibration}
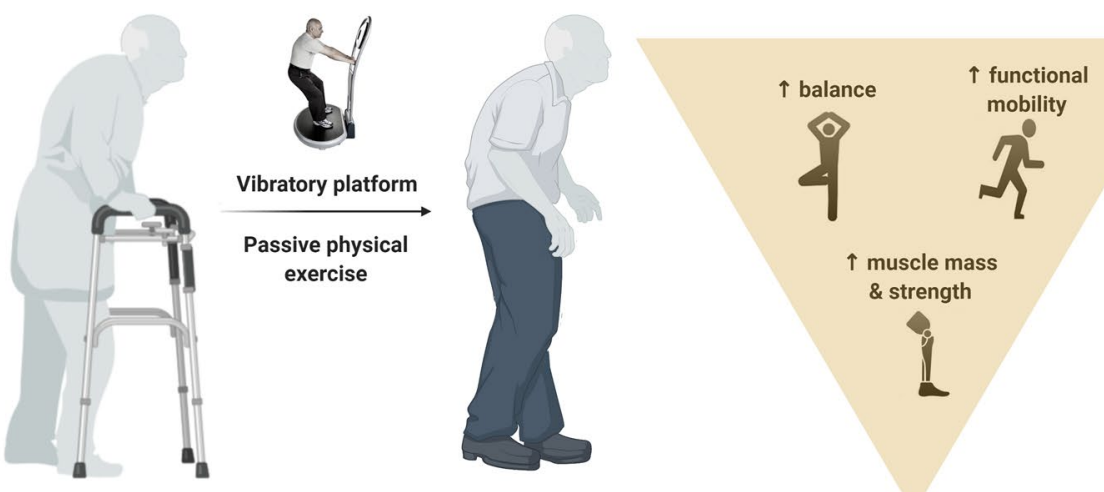

\section{Transcranial Ultrasound Stimulation}
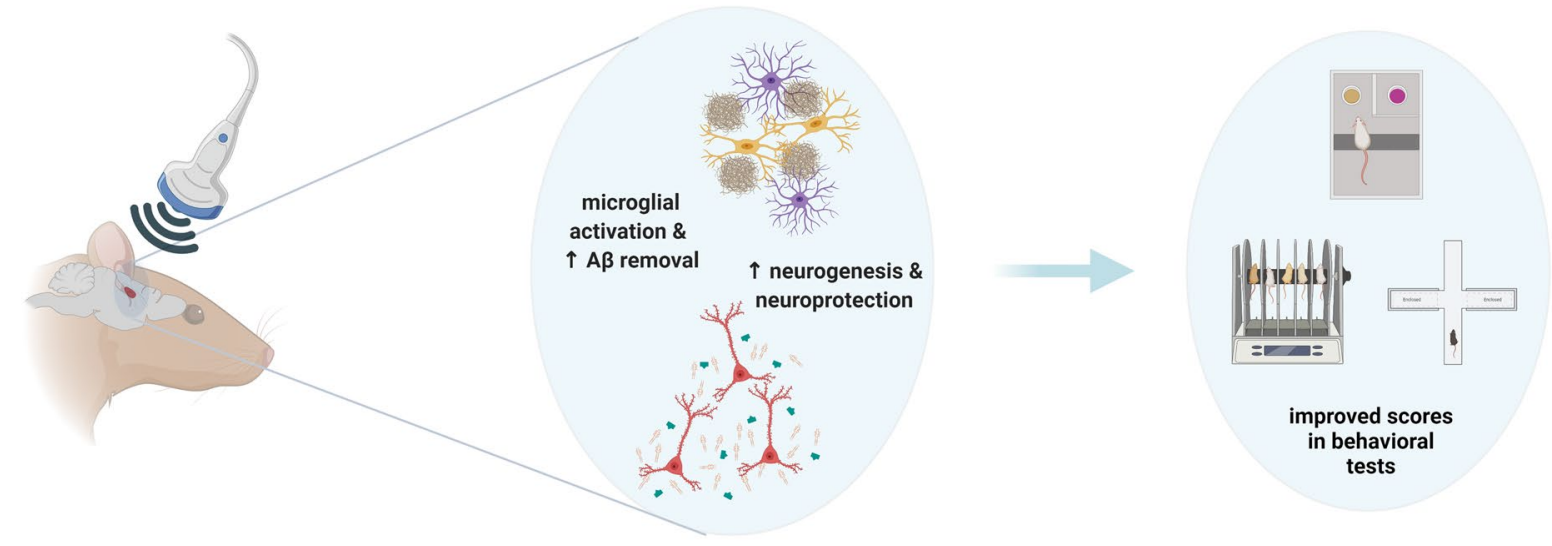

Auditory Stimulation

40- $\mathrm{Hz}$ sound
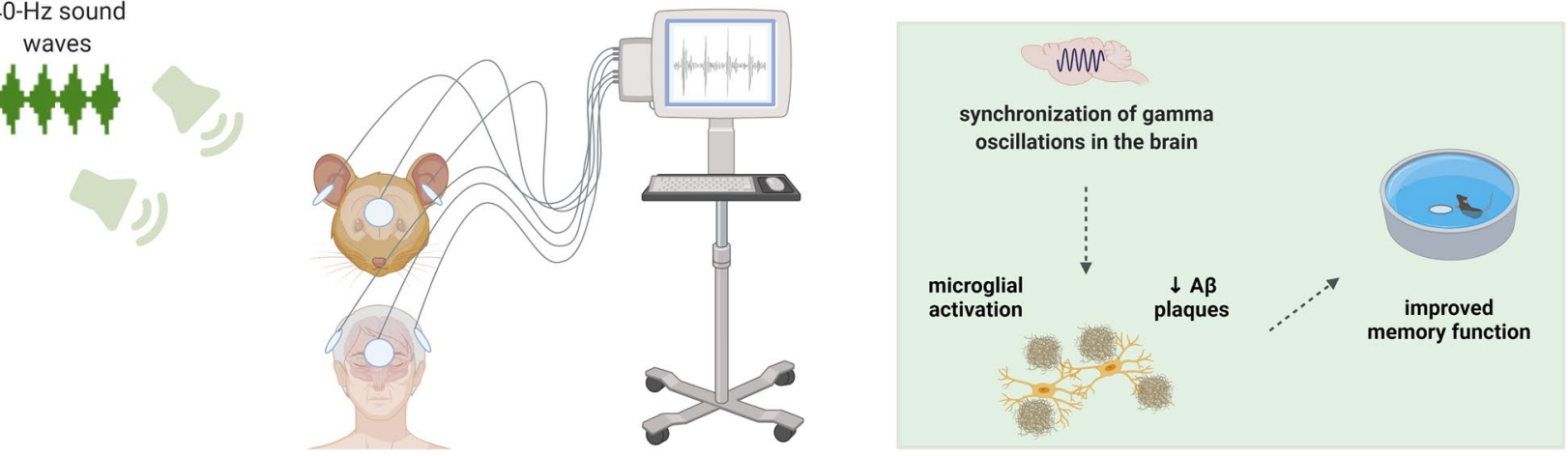

Fig. 2 Schematic representation of the main mechanical vibration effects on AD pathology and age-related impairments. Created with BioRender. com 


\section{Abbreviations}

A 3 : Amyloid- $\beta$; AD: Alzheimer's disease; APP: Amyloid precursor protein; AS: Auditory stimulation; BACE-1: Beta secretase-1; BBB: Blood-brain barrier; EEG: Electroencephalography; EPHPP: Effective Public Health Practice Project; MCI: Mild cognitive impairment; PET: Positron emission tomography; PRISMA: Preferred Reporting Items for Systematic Reviews and Meta-Analyses; STAIR: Stroke Therapy Academic Industry Roundtable; TUSS: Transcranial ultrasound stimulation; US: Ultrasound; WBV: Whole-body vibration.

\section{Supplementary Information}

The online version contains supplementary material available at https://doi. org/10.1186/s40035-021-00256-z.

Additional file 1. Table S1. Quality assessment data for clinical trials, using the EPHPP Quality Assessment Tool. Table S2. Quality assessment data for animal studies, using the STAIR preclinical recommendations.

\section{Acknowledgements}

Not applicable.

\section{Authors' contributions}

All authors contributed to conceiving the context of this systematic review. The literature review, study selection, and initial manuscript writing were done by FM and IS, while FS, IS, OC and NS provided critical feedback on the manuscript. FM, FS and IS wrote and finalized the manuscript. All authors read and approved the final manuscript.

\section{Funding}

This systematic review was supported by FCT (Fundação para a Ciência e Tecnologia) through the grant with reference SFRH/BD/09375/2020, and in the scope of the projects UIDB/04436/2020, UIDP/04436/2020, and NORTE-010145-FEDER-000023, funded by the European Fund for Regional Development (FEDER) of the Operational Programme for Competitiveness and Internationalization (POCI), by Portugal 2020

\section{Availability of data and materials}

Not applicable.

\section{Declarations}

Ethics approval and consent to participate

Not applicable.

\section{Consent for publication}

Not applicable.

\section{Competing interests}

The authors declare that they have no competing interests.

\section{Author details}

${ }^{1}$ Center for Microelectromechanical Systems (CMEMS), University of Minho, Campus Azurém, 4800-058 Guimarães, Portugal. ${ }^{2}$ Life and Health Sciences Research Institute (ICVS), Medical School, University of Minho, Campus Gualtar, 4710-057 Braga, Portugal. ${ }^{3}$ ICVS/3B's - PT Government Associate Laboratory, Braga/Guimarães, Portugal. ${ }^{4}$ Institute of Biosciences \& Applications NCSR "Demokritos", Athens, Greece.

Received: 5 March 2021 Accepted: 3 August 2021

Published online: 24 September 2021

\section{References}

1. Murman DL. The impact of age on cognition. Semin Hear. 2015;36(3):111-21.

2. Castano-Prat P, Perez-Mendez L, Perez-Zabalza M, Sanfeliu C, GiménezLlort L, Sanchez-Vives MV. Altered slow $(<1 \mathrm{~Hz})$ and fast (beta and gamma) neocortical oscillations in the 3xTg-AD mouse model of Alzheimer's disease under anesthesia. Neurobiol Aging. 2019;79:142-51.

3. Nichols E, Szoeke CEl, Vollset SE, Abbasi N, Abd-Allah F, Abdela J, et al. Global, regional, and national burden of Alzheimer's disease and other dementias, 1990-2016: a systematic analysis for the Global Burden of Disease Study 2016. Lancet Neurol. 2018;18(1):88-106.

4. Palmqvist S, Schöll M, Strandberg O, Mattsson N, Stomrud E, Zetterberg $\mathrm{H}$, et al. Earliest accumulation of $\beta$-amyloid occurs within the defaultmode network and concurrently affects brain connectivity. Nat Commun. 2017:8(1):1-13.

5. Amemori T, Jendelova P, Ruzicka J, Urdzikova LM, Sykova E. Alzheimer's disease: Mechanism and approach to cell therapy. Int J Mol Sci. 2015;16(11):26417-51.

6. Querfurth HW, Laferla FM. Mechanisms of disease. N Engl J Med. 2010;362(4):329-44.

7. World Health Organization. Risk reduction of cognitive decline and dementia: WHO guidelines (2019). https://www.who.int/ mental_health/neurology/dementia/en.

8. World Health Organization. (2017). Global action plan on the public health response to dementia 2017-2025. https://www.who.int/ mental_health/neurology/dementia/en

9. Huang LK, Chao SP, Hu CJ. Clinical trials of new drugs for Alzheimer disease. J Biomed Sci. 2020;27(1):18.

10. Johnstone DM, Moro C, Stone J, Benabid AL, Mitrofanis J. Turning on lights to stop neurodegeneration: the potential of near infrared light therapy in Alzheimer's and Parkinson's disease. Front Neurosci. 2016;9:500.

11. Rajji TK. Transcranial magnetic and electrical stimulation in Alzheimer's disease and mild cognitive impairment: a review of randomized controlled trials. Clin Pharmacol Ther. 2019;106(4):776-80.

12. Guo T, Ren P, Hao S, Wang B. The underestimated role of mechanical stimuli in brain diseases and the related in vitro models. Curr Pharm Des. 2017;23(15):2161-2176.

13. Lam FMHH, Lau RWKK, Chung RCKK, Pang MYCC. The effect of whole body vibration on balance, mobility and falls in older adults: a systematic review and meta-analysis. Maturitas. 2012;72(3):206-13.

14. Merriman $\mathrm{H}$, Jackson $\mathrm{K}$. The effects of whole-body vibration training in aging adults: a systematic review. J Geriatr Phys Ther. 2009;32(3):134-45.

15. Blankevoort CG, Van HJG, Boersma F. Review of effects of physical activity on strength, balance, mobility and ADL performance in elderly subjects with dementia. Dement Geriatr Cogn Disord. 2010;30:392-402.

16. Bautmans I, Van Hees E, Lemper JC, Mets T. The feasibility of whole body vibration in institutionalised elderly persons and its influence on muscle performance, balance and mobility: a randomised controlled trial [ISRCTN62535013]. BMC Geriatr. 2005;5:1-8.

17. Verschueren SMP, Roelants M, Delecluse C, Swinnen S, Vanderschueren D, Boonen S. Effect of 6-month whole body vibration training on hip density, muscle strength, and postural control in postmenopausal women: a randomized controlled pilot study. J Bone Miner Res. 2004;19(3):352-9.

18. Lai C, Tseng S, Liu B, Lee M, Chen H. Effect of whole-body vibration for 3 months on arterial stiffness in the middle-aged and elderly. Clin Interv Aging. 2014;9:821-8.

19. Haas CT, Turbanski S, Kessler K, Schmidtbleicher D. The effects of random whole-body-vibration on motor symptoms in Parkinson 's disease. NeuroRehabilitation. 2006;21:29-36.

20. Lee GC. Does whole-body vibration training in the horizontal direction have effects on motor function and balance of chronic stroke survivors? A preliminary study. J Phys Ther Sci. 2015;27(4):1133-6.

21. Lam F, Liao R, Kwok T, Pang M. Effects of adding whole-body vibration to routine day activity program on physical functioning in elderly with mild or moderate dementia : a randomized controlled trial. Int J Geriatr Psychiatry. 2017;33(1):21-30.

22. Lee SJ, Park KW, Kim LS, Kim H. Effects of noise level and cognitive function on speech perception in normal elderly and elderly with amnestic mild cognitive impairment. Cogn Behav Neurol. 2016;29(2):68-77.

23. Yoo SS, Bystritsky A, Lee JH, Zhang Y, Fischer K, Min BK, et al. Focused ultrasound modulates region-specific brain activity. Neuroimage. 2011;56(3):1267-75 
24. Tufail Y, Matyushov A, Baldwin N, Tauchmann ML, Georges J, Yoshihiro A, et al. Transcranial pulsed ultrasound stimulates intact brain circuits. Neuron. 2010;66(5):681-94.

25. Mooney SJ, Shah K, Yeung S, Burgess A, Aubert I, Hynynen K. Focused ultrasound-induced neurogenesis requires an increase in blood-brain barrier permeability. PLoS One. 2016;11(7):1-11.

26. Chu PC, Liu HL, Lai HY, Lin CY, Tsai HC, Pei YC. Neuromodulation accompanying focused ultrasound-induced blood-brain barrier opening. Sci Rep. 2015;5:1-12.

27. Kovacs ZI, Tu TW, Sundby M, Qureshi F, Lewis BK, Jikaria N, et al. MRI and histological evaluation of pulsed focused ultrasound and microbubbles treatment effects in the brain. Theranostics. 2018:8(17):4837-55.

28. Hatch RJRJ, Leinenga G, Götz J. Scanning ultrasound (SUS) causes no changes to neuronal excitability and prevents age-related reductions in hippocampal CA1 dendritic structure in wild-type mice. PLoS One. 2016;11(10):1-14

29. Vaezzadeh M, Saeidi M, Firouzkhani A, Abdolmajidi B, Hajnorouzi A. Alzheimer treatment by applying ultrasound waves. Curr Signal Transduct Ther. 2011;6(3):424-7.

30. Martorell AJ, Paulson AL, Suk HJ, Abdurrob F, Drummond GT, Guan W, et al. Multi-sensory gamma stimulation ameliorates Alzheimer's-associated pathology and improves cognition. Cell. 2019;177(2):256-271.e22.

31. Papalambros NA, Weintraub S, Chen T, Grimaldi D, Santostasi G, Paller $K A$, et al. Acoustic enhancement of sleep slow oscillations in mild cognitive impairment. Ann Clin Transl Neurol. 2019;6(7):1191-201.

32. Ekert JO, Gould RL, Reynolds G, Howard RJ. TNF alpha inhibitors in alzheimer's disease: a systematic review. Int J Geriatr Psychiatry. 2018;33(5):688-94.

33. Landeiro F, Mughal S, Walsh K, Nye E, Morton J, Williams H, et al. Healthrelated quality of life in people with predementia Alzheimer's disease, mild cognitive impairment or dementia measured with preferencebased instruments: a systematic literature review. Alzheimers Res Ther. 2020;12(1):1-14.

34. Jackson N, Waters E. Criteria for the systematic review of health promotion and public health interventions. Health Promot Int. 2005;20(4):367-74.

35. Fisher M, Feuerstein G, Howells DW, Hurn PD, Kent TA, Savitz SI, et al. Update of the stroke therapy academic industry roundtable preclinical recommendations. Stroke. 2009;40(6):2244-50.

36. Leinenga G, Götz J. Scanning ultrasound removes amyloid- $\beta$ and restores memory in an Alzheimer's disease mouse model. Sci Trans Med. 2015:7(278):278ra33.

37. Poon CT, Shah K, Lin C, Tse R, Kim KK, Mooney S, et al. Time course of focused ultrasound effects on $\beta$-amyloid plaque pathology in the TgCRND8 mouse model of Alzheimer's disease. Sci Rep. 2018;8(1):1-11.

38. Burgess A, Dubey S, Yeung S, Hough O, Eterman N, Aubert I, et al. Alzheimer disease in a mouse model: MR imaging-guided focused ultrasound targeted to the hippocampus opens the blood-brain barrier and improves pathologic abnormalities and behavior. Radiology. 2014;273(3):736-45

39. Cardinale M, Bosco C. The use of vibration as an exercise intervention. Exerc Sport Sci Rev. 2003;31:3-7.

40. Torvinen $S$, Kannus $P$, Sievänen $H$, Järvinen TA, Pasanen M, Kontulainen $S$, et al. Effect of four-month vertical whole body vibration on performance and balance. Med Sci Sports Exerc. 2002;34(9):1523-8.

41. Todd N, Zhang Y, Arcaro M, Becerra L, Borsook D, Livingstone M, et al. Focused ultrasound induced opening of the blood-brain barrier disrupts inter-hemispheric resting state functional connectivity in the rat brain. Neuroimage. 2018;178:414-22.

42. Xhima K, Markham-Coultes K, Nedev H, Heinen S, Saragovi HU, Hynynen $K$, et al. Focused ultrasound delivery of a selective TrkA agonist rescues cholinergic function in a mouse model of Alzheimer's disease. Sci Adv. 2020;6(4):eaax6646.

43. Janowicz PWPW, Leinenga G, Götz J, Nisbet RM. Ultrasound-mediated blood-brain barrier opening enhances delivery of therapeutically relevant formats of a tau-specific antibody. Sci Rep. 2019;9(1):1-9.

44. Chang EL, Ting CY, Hsu PH, Lin YC, Liao EC, Huang CY, et al. Angiogenesis-targeting microbubbles combined with ultrasound-mediated gene therapy in brain tumors. J Control Release. 2017;255:164-75.

45. Karakatsani MEME, Kugelman T, Ji R, Murillo M, Wang S, Niimi Y, et al. Unilateral focused ultrasound-induced blood-brain barrier opening reduces phosphorylated Tau from the rTg4510 mouse model. Theranostics. 2019;9(18):5396-411.

46. Hsu PH, Lin YT, Chung YH, Lin KJ, Yang LY, Yen TC, et al. focused ultrasound-induced blood-brain barrier opening enhances GSK-3 inhibitor delivery for amyloid-beta plaque reduction. Sci Rep. 2018;8(1):12882.

47. Nisbet RM, Van der Jeugd A, Leinenga G, Evans HT, Janowicz PW, Götz J. Combined effects of scanning ultrasound and a tau-specific single chain antibody in a tau transgenic mouse model. Brain. 2017:140(5):1220-30.

48. Lee J, Ryu S, Kim HJ, Jung J, Lee B, Kim T. 40 Hz acoustic stimulation decreases amyloid beta and modulates brain rhythms in a mouse model of Alzheimer's disease. bioRxiv. 2018. https://doi.org/10.1101/ 390302.

49. Calomeni MR, Furtado da Silva V, Velasques BB, Feijó OG, Bittencourt JM Ribeiro de Souza E Silva AP. Modulatory effect of association of brain stimulation by light and binaural beats in specific brain waves. Clin Pract Epidemiol Ment Health. 2017;13(1):134-44.

50. Palop JJ, Mucke L. Network abnormalities and interneuron dysfunction in Alzheimer disease. Nat Rev Neurosci. 2016;17(12):777-92.

51. laccarino HF, Singer AC, Martorell AJ, Rudenko A, Gao F, Gillingham TZ, et al. Gamma frequency entrainment attenuates amyloid load and modifies microglia. Nature. 2016;540(7632):230-5.

52. Başar E. A review of gamma oscillations in healthy subjects and in cognitive impairment. Int J Psychophysiol. 2013;90(2):99-117.

53. Gillespie AK, Jones EA, Lin YH, Karlsson MP, Kay K, Yoon SY, et al. Apolipoprotein E4 causes age-dependent disruption of slow gamma oscillations during hippocampal sharp-wave ripples. Neuron. 2016;90(4):740-51.

54. Adaikkan C, Middleton SJ, Marco A, Pao PC, Mathys H, Kim DNW, et al. Gamma entrainment binds higher-order brain regions and offers neuroprotection. Neuron. 2019;102(5):929-43.

55. Ngo HV, Martinetz T, Born J, Mölle M. Auditory closed-loop stimulation of the sleep slow oscillation enhances memory. Neuron. 2013;78(3):545-53.

56. Lee Y, Choi Y, Park EJ, Kwon S, Kim H, Lee JY, et al. Improvement of glymphatic-lymphatic drainage of beta-amyloid by focused ultrasound in Alzheimer's disease model. Sci Rep. 2020;10(1):1-14.

57. Eguchi K, Shindo T, Ito K, Ogata T, Kurosawa R, Kagaya Y, et al. Wholebrain low-intensity pulsed ultrasound therapy markedly improves cognitive dysfunctions in mouse models of dementia-crucial roles of endothelial nitric oxide synthase. Brain Stimul. 2018;11(5):959-73.

58. Shen Y, Hua L, Yeh C-KC, Shen L, Ying M, Zhang Z, et al. Ultrasound with microbubbles improves memory, ameliorates pathology and modulates hippocampal proteomic changes in a triple transgenic mouse model of alzheimer's disease. Theranostics. 2020;10(25):11794-819.

59. Jordão JF, Thévenot E, Markham-Coultes K, Scarcelli T, Weng YQ, Xhima $K$, et al. Amyloid- $\beta$ plaque reduction, endogenous antibody delivery and glial activation by brain-targeted, transcranial focused ultrasound. Exp Neurol. 2013;248:16-29.

60. Leinenga G, Götz J. Safety and efficacy of scanning ultrasound treatment of aged APP23 mice. Front Neurosci. 2018;12:1-10.

61. Bobola MS, Chen L, Ezeokeke CK, Olmstead TA, Nguyen C, Sahota A, et al. Transcranial focused ultrasound, pulsed at $40 \mathrm{~Hz}$, activates microglia acutely and reduces $A \beta$ load chronically, as demonstrated in vivo. Brain Stimul. 2020;13(4):1014-23.

62. O'Reilly MA, Jones RMRM, Barrett E, Schwab A, Head E, Hynynen K. Investigation of the safety of focused ultrasound-induced blood-brain barrier opening in a natural canine model of aging. Theranostics. 2017;7(14):3573-84.

63. Lipsman N, Meng Y, Bethune AJAJ, Huang Y, Lam B, Masellis M, et al. Blood-brain barrier opening in Alzheimer's disease using MR-guided focused ultrasound. Nat Commun. 2018;9(1):1-8.

64. D'Haese PF, Ranjan M, Song A, Haut MW, Carpenter J, Dieb G, et al. $\beta$-Amyloid plaque reduction in the hippocampus after focused ultrasound-induced blood-brain barrier opening in Alzheimer's disease. Front Hum Neurosci. 2020;14:593672.

65. D'Haese PF, Ranjan M, Song A, Haut MW, Carpenter J, Dieb G, et al. $\beta$-Amyloid plaque reduction in the hippocampus after focused ultrasound-induced blood-brain barrier opening in Alzheimer's disease. Front Hum Neurosci. 2020;14:593672. 
66. Pandit R, Leinenga G, Götz J. Repeated ultrasound treatment of tau transgenic mice clears neuronal tau by autophagy and improves behavioral functions. Theranostics. 2019;9(13):3754-67.

67. Kim KH, Lee HB. The effects of whole body vibration exercise intervention on electroencephalogram activation and cognitive function in women with senile dementia. J Exerc Rehabil. 2018;14(4):586-91.

68. Meng Y, Maclntosh BJBJ, Shirzadi Z, Kiss A, Bethune A, Heyn C, et al. Resting state functional connectivity changes after MR-guided focused ultrasound mediated blood-brain barrier opening in patients with Alzheimer's disease. Neuroimage. 2019;200:275-80.

69. Beisteiner R, Matt E, Fan C, Baldysiak H, Schönfeld M, Philippi Novak T, et al. Transcranial pulse stimulation with ultrasound in Alzheimer's disease-a new navigated focal brain therapy. Adv Sci. 2020;7(3):1902583.

70. Papalambros NA, Santostasi G, Malkani RG, Braun R, Weintraub S, Paller $K A$, et al. Acoustic enhancement of sleep slow oscillations and concomitant memory improvement in older adults. Front Hum Neurosci. 2017;11:1-14

71. Busse A, Angermeyer MC, Riedel-Heller SG. Progression of mild cognitive impairment to dementia: a challenge to current thinking. $\mathrm{Br} J$ Psychiatry. 2006;189:399-404.

72. Zhu Y, Peng N, Zhou M, Liu P, Qi X, Wang N, et al. Tai Chi and wholebody vibrating therapy in sarcopenic men in advanced old age: a clinical randomized controlled trial. Eur J Ageing. 2019;16(3):273-82

73. Furness T, Maschette W. Influence of whole body vibration platform frequency on neurmuscular performance of community-dwelling older adults. J Strength Cond Res. 2009;23(5):1508-13.

74. Cheung WH, Mok HW, Qin L, Sze PC, Lee KM, Leung KS. High-frequency whole-body vibration improves balancing ability in elderly women. Arch Phys Med Rehabil. 2007;88(7):852-7.

75. Kawanabe K, Kawashima A, Sashimoto I, Takeda T, Sato Y, Iwamoto J. Effect of whole-body vibration exercise and muscle strengthening, balance, and walking exercises on walking ability in the elderly. Keio J Med. 2007:56(1):28-33.

76. Zhang L, Weng C, Liu M, Wang Q, Liu L, He Y. Effect of whole-body vibration exercise on mobility, balance ability and general health status in frail elderly patients: a pilot randomized controlled trial. Clin Rehabil. 2014;28(1):59-68

77. Machado A, García-López D, González-Gallego J, Garatachea N, GarciaLopéz D, González-Gallego J, et al. Whole-body vibration training increases muscle strength and mass in older women: a randomizedcontrolled trial. Scand J Med Sci Sport. 2010;20(2):200-7.

78. Álvarez-Barbosa F, Del Pozo-Cruz J, Del Pozo-Cruz B, Alfonso-Rosa RM, Rogers ME, Zhang Y. Effects of supervised whole body vibration exercise on fall risk factors, functional dependence and healthrelated quality of life in nursing home residents aged $80+$. Maturitas. 2014;79(4):456-63.

79. Marín PJ, Martín-lópez A, Vicente-campos D, García-pastor T, Garatachea $\mathrm{N}$, Chicharro JL, et al. Effects of vibration training and detraining on balance and muscle strength in older adults. J Sport Sci Med. 2011;10(3):559-64.

80. Logsdon RG, Gibbons LE, McCurry SM, Teri L. Assessing quality of life in older adults with cognitive impairment. Psychosom Med. 2002;64(3):510-9.

81. Sievänen H, Karinkanta S, Moisio-Vilenius P, Ripsaluoma J. Feasibility of whole-body vibration training in nursing home residents with low physical function: a pilot study. Aging Clin Exp Res. 2014;26(5):511-7.

82. Rezai AR, Ranjan M, D'Haese PF, Haut MW, Carpenter J, Najib U, et al. Noninvasive hippocampal blood-brain barrier opening in Alzheimer's disease with focused ultrasound. Proc Natl Acad Sci U S A 2020;117(17):9180-2.

83. Clements-Cortes A, Ahonen H, Evans M, Freedman M, Bartel L. Shortterm effects of rhythmic sensory stimulation in Alzheimer's disease: an exploratory pilot study. J Alzheimers Dis. 2016;52(2):651-60.

84. Heesterbeek M, Van Der Zee EA, Van Heuvelen MJG, Anton van der Zee E, Joan Gerda van Heuvelen M, Van Der Zee EA, et al. Feasibility of three novel forms of passive exercise in a multisensory environment in vulnerable institutionalized older adults with dementia. J Alzheimers Dis. 2019;70(3):681-90.

85. Downs ME, Buch A, Sierra C, Karakatsani ME, Teichert T, Chen S, et al. Correction: long-term safety of repeated blood-brain barrier opening via focused ultrasound with microbubbles in non-human primates performing a cognitive task. PLoS One. 2015;10(6):e0130860.

86. O'Reilly MA, Hynynen K. Blood-brain barrier: real-time feedback-controlled focused ultrasound disruption by using an acoustic emissionsbased controller. Radiology. 2012;263(1):96-106.

87. Fuermaier ABM, Tucha L, Koerts J, Van Heuvelen MJG, Van Der Zee EA, Lange KW, et al. Good vibrations-effects of whole body vibration on attention in healthy individuals and individuals with ADHD. PLoS One. 2014;9(2):e90947.

88. Regterschot GRH, Van Heuvelen MJGG, Zeinstra EB, Fuermaier ABMM, Tucha L, Koerts J, et al. Whole body vibration improves cognition in healthy young adults. PLoS One. 2014;9(6):e100506.

89. Milanese C, Piscitelli F, Simoni C, Pugliarello R, Zancanaro C. Effects of whole-body vibration with or without localized radiofrequency on anthropometry, body composition, and motor performance in young nonobese women. J Altern Complement Med. 2012;18(1):69-75.

90. Cochrane DJ, Stannard SR. Acute whole body vibration training increases vertical jump and flexibility performance in elite female field hockey players. Br J Sports Med. 2005;39(11):860-5.

91. Bogaerts A, Verschueren S, Delecluse C, Claessens AL, Boonen S. Effects of whole body vibration training on postural control in older individuals: a 1 year randomized controlled trial. Gait Posture. 2007;26:309-16.

92. Gusi N, Raimundo A, Leal A. Low-frequency vibratory exercise reduces the risk of bone fracture more than walking: a randomized controlled trial. BMC Musculoskelet Disord. 2006;7(92):1-8.

93. Pollock RD, Martin FC, Newham DJ. Whole-body vibration in addition to strength and balance exercise for falls-related functional mobility of frail older adults: a single-blind randomized controlled trial. Clin Rehabil. 2012;26(10):915-23.

94. Wilcock DM, DiCarlo G, Henderson D, Jackson J, Clarke K, Ugen KE, et al. Intracranially administered anti-A $\beta$ antibodies reduce $\beta$-amyloid deposition by mechanisms both independent of and associated with microglial activation. J Neurosci. 2003;23(9):3745-51.

95. Holmes C, Boche D, Wilkinson D, Yadegarfar G, Hopkins V, Bayer A, et al. Long-term effects of A 42 immunisation in Alzheimer's disease: follow-up of a randomised, placebo-controlled phase I trial. Lancet. 2008;372(9634):216-23.

96. Cirrito JR, Yamada KA, Finn MB, Sloviter RS, Bales KR, May PC, et al. Synaptic activity regulates interstitial fluid amyloid- $\beta$ levels in vivo. Neuron. 2005:48(6):913-22.

97. Mille M, Johnson ME, Martinez KM, Rogers MW. Age-dependent differences in lateral balance recovery through protective stepping. Clin Biomech. 2005;20:607-16.

98. Garatachea N, Jlménez A, Bresciani G, Mariño NA, González-Gallego J, De Paz JA. The effects of movement velocity during squatting on energy expenditure and substrate utilization in whole-body vibration. J Strength Cond Res. 2007;21(2):594-8.

99. Torvinen S, Kannus P, Sievänen H, Järvinen TAH, Pasanen M, Kontulainen $\mathrm{S}$, et al. Effect of 8-month vertical whole body vibration on bone, muscle performance, and body balance: a randomized controlled study. J Bone Miner Res. 2003;18(5):876-84.

100. Cardinale M, Soiza RL, Leiper JB, Gibson A, Primrose WR. Hormonal responses to a single session of whole- body vibration exercise in older individuals. Br J Sports Med. 2010;44(4):284-8.

101. Moher D, Liberati A, Tetzlaff J, Altman DGG, Altman DGG, Antes G, et al. Preferred reporting items for systematic reviews and metaanalyses: the PRISMA statement. Ann Intern Med. 2009;151(4):264-9.

102. Beaudart C, Maquet D, Mannarino M, Buckinx F, Demonceau M, Crielaard JM, et al. Effects of 3 months of short sessions of controlled whole body vibrations on the risk of falls among nursing home residents. BMC Geriatr. 2013;13:42. 